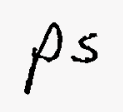

PPPL-3154 - Preprint Date: January 1996, UC-420, 426,

\section{Search for Alpha-Driven TAE Modes at Lowered Ion Temperature in TFTR DT Discharges}

\author{
S. J. Zweben, R.V. Budny, C.Z. Cheng, E.D. Fredrickson, G. Y. Fu, \\ D. R. Mikkelsen, G. L. Schmidt, S. D. Scott, D.A. Spong $\ddagger$, C.E. Bush, Z. Chang, D.S. \\ Darrow, R. Fonck*, L. Grisham, E. Mazzucato, R. Nazikian, \\ D.K. Owens, H. Park, S. Paul, J. Schivell, J.D. Strachan, E. Synakowski, \\ G. Taylor, and K. M. Young
}

Princeton Plasma Physics Laboratory, P.O. Box 451, Princeton, N.J. 08543

\$ Oak Ridge National Laboratory, Oak Ridge, Tenn. 37831

* University of Wisconsin, Madison, Wisc. 53706

\begin{abstract}
An experiment was performed in TFTR DT plasmas to attempt to destabilize the alpha particle driven Toroidicity-induced Alfven Eigenmode (TAE) by transiently cooling the ions, which should have lowered the ion Landau damping of the TAE modes. Transient cooling perturbations were made during the NBI heating phase of high powered DT supershots using $\mathrm{He}$ gas puffs or deuterium $(\mathrm{D})$ or lithium $(\mathrm{Li})$ pellet injection. The ion temperature was successfully lowered from $T_{i}(0) \approx 20 \mathrm{keV}$ to $T_{i}(0) \approx 10 \mathrm{keV}$ in about $0.2 \mathrm{sec}$; however, no signs of alpha-driven TAE modes were observed. Theoretical analyses of these discharges suggested that the alpha pressure required for TAE instability was about a factor of 2-3 greater than actually obtained in this experiment, consistent with the absence of alpha-driven TAE modes.
\end{abstract}

\title{
MASTER
}

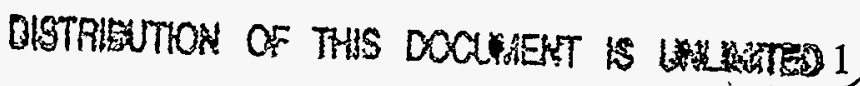




\section{Introduction}

One of the main issues for ignited fusion reactors concerns potential alpha particle driven instabilities such as the Toroidal Alfven Eigenmodes (TAE). The TAE mode is one type of global shear-Alfven MHD eigenmode in a torus, which in theory can be driven unstable by the free energy associated with a sufficiently large pressure gradient of super-Alfvenic alpha particles[1-4].

Previous tokamak experiments in deuterium (DD) plasmas have studied TAE modes driven unstable using fast ions from neutral beam injection (NBI)[5-6] or ion cyclotron minority heating (ICRH)[7-8]. These studies have been extended in TFTR to DT alpha particles, which have a higher energy and a more isotropic distribution function than either the NBI or ICRH fast ions. Although the alpha particle pressure in TFTR is $\approx 5-10$ times smaller than the fast ion pressures obtained with NBI or ICRH, the alpha pressure gradient in TFTR is within about a factor of 2-3 of that expected for future alpha-driven DT tokamak reactors such as ITER[9,10].

The alpha particle pressure in TFTR is generally maximized along with the DT fusion power, since the alpha particle thermalization time is roughly independent of plasma conditions in the high ion temperature "supershot" regime. However, so far no clear signs of alpha-driven TAE modes have been observed in standard DT supershots with up to 10.7 MW of fusion power[11-14].

The present experiment was motivated by an analysis of TAE stability in TFTR DT[1], which suggested that the dominant TAE damping mechanism is ion Landau damping, which can be reduced by lowering the ion temperature or ion beta $\left(J_{i}\right)$. The experiment was designed to reduce the ion temperature while maintaining the maximum possible alpha particle pressure. This was accomplished by transiently cooling a high fusion power supershot (similar to that described previously in Ref. 11) by adding $\mathrm{He}$ gas puffs or $\mathrm{D}$ or Li pellet injection.

The result of this experiment is that no signs of alpha-driven TAE modes were observed even though the ion temperature was lowered from $T_{i}(0) \approx 20 \mathrm{keV}$ to $T_{i}(0) \approx 10$ $\mathrm{keV}$ in about $0.2 \mathrm{sec}$. Theoretical analyses of the TAE instability of these DT discharges indicate that the alpha pressure required for TAE stability remained about a factor of 23 greater than actually obtained in this experiment, partly due to a reduction in the alpha particle beta during these cooling perturbations. Thus the theoretical results are consistent with the absence of alpha-driven TAE modes in this experiment.

The outline of this paper is as follows: the theoretical motivation is described in Sec. 2, the experimental scenarios are discussed sec. 3 , the expental results are presented in Sec. 4, the TAE stability analyses are presented in Sêc. 5 , and a Discussion is in Sec. 6, including a summary and suggestions for further work. 


\section{DISCLAIMER}

Portions of this document may be illegible in electronic image products. Images are produced from the best available original document. 


\section{Theoretical Motivations}

The first analysis of alpha-driven TAE modes in a tokamak determined the instability threshold by equating the "drive" from the local super-Alfvenic alpha particle pressure gradient with the local parallel electron damping[15]. It was soon recognized that this local $\int a$ threshold for TAE instability would be exceeded in the TFTR DT experiments[16].

Subsequently, increasingly realistic calculations for alpha-driven TAE modes in TFTR DT plasmas have been made by including the 2-dimensional eigenmode geometry, the finite alpha orbit widths and gyroradii, and additional damping mechanisms such as ion Landau damping and continuum damping. In general, these added effects have been in the stabilizing direction, so that alpha-driven TAE modes are now calculated to be stable in the highest powered TFTR DT discharges[2,3,12-14].

Amajor reason for this increased TAE stability is the inclusion of ion Landau damping, which can be relatively large in TFTR due to the very high ion temperature in the supershot regime. Ion Landau damping of TAE modes in tokamaks is mainly due to the poloidally varying magnetic drift velocity[15], which creates a new resonance at $1 / 3$ of the Alfven speed, which is near the speed of thermal or beam ions in TFTR (Table 1). Although in general there can be either driving or damping from the ion distribution functions, in TFTR supershots the thermal and beam ions damp the TAE mode, while the alpha particles tend to drive it. The thermal and beam ion damping is particularly important in TFTR since it depends on the pressure of these ions, which is $\geq 5$ times the alpha particle pressure (Table 1).

A simple expression for the thermal ion Landau damping rate $\odot_{\mathrm{i}}$, normalized to the TAE frequency $\Sigma_{0}$, for a Maxwellian ion species is[17]:

$$
\Theta_{i} / \Sigma_{0} U \dot{U} 7 \times 10 \quad-3 q^{2} \int_{i}^{-3 / 2} \exp \left(-1 /\left[9 \quad \int_{i}\right]\right)
$$

where $\int_{i}$ is the ion beta, which depends on both the ion temperature and ion density. Therefore the thermal ion Landau damping should decrease to a negligible level if the central ion beta is reduced by a factor of two from its normal supershot value of $\int_{\mathfrak{a}}(0) \approx 0.017 \%$, as illustrated in Fig. 1 .

Several detailed numerical studies of TFTR TAE stability were made prior the DT run, based on extrapolating a standard I=1.6 MA deuterium-only NBI (DD) supershot to deuterium-tritium NBI (DT) using TRANSP[18]. For these preliminary analyses the ion population was simulated by a single-species Maxwellian at the measured TFTR ion temperature of $T_{i}(0) \approx 20 \mathrm{keV}$, i.e. no separate beam ion component was included, and the plasma parameters and profiles were taken to be the $\mathrm{DD}$ experimental values. Both the 
NOVA-K[1] and the TAE/FL[19] codes suggested that ion Landau damping would be the dominant damping mechanism for low- $n$ modes in TFTR supershots $(n=1-5)$. However, the calculated threshold for alpha-driven TAE modes was still a factor of 2-3 higher than the expected experimental alpha pressure, i.e. the alpha-driven TAE modes were predicted to be stable, largely due to the effect of ion Landau damping.

The present experiment was motivated by these early theoretical studies, which suggested that the alpha-driven TAE might be destabilized by reducing the ion Landau damping in TFTR DT supershots. In TFTR, the ion Landau damping can most easily be reduced by lowering the ion temperature, since the density cannot easily be lowered while maintaining a high alpha pressure, and the magnetic field could not be raised very much.

Additional TAE simulations were performed to determine the effect of lowering the ion temperature in a standard DT supershot. The NOVA-K code simulated the effect of reducing the ion temperature using a scan of NBI heating power, self-consistently varying the other parameters such as electron temperature, density, and alpha particle beta. The result was that the TAE was predicted to be marginally unstable at an ion temperature of $\mathrm{T}_{\mathrm{i}}(0) \approx 10 \mathrm{keV}[1]$. The TAE/FL code varied the ion temperature while holding all profiles and other parameters fixed, with the result that the total TAE damping rate was decreased by about a factor of 2 between $T_{i}(0) \approx 20 \mathrm{keV}$ and $10 \mathrm{keV}[19]$. However, these calculations were sensitive to the plasma profiles, e.g. through the radial variation of the TAE mode structure, alpha drive, and damping rates. For example, the sensitivity of the TAE growth rate to $\mathrm{T}_{\mathbf{i}}(0)$ was found to be much reduced for an $\mathrm{Ip}=1.8 \mathrm{MA}$ case with a slightly different equilibrium than the original Ip=1.6 MA case [19].

Another physics motivation for this experiment is the theoretical observation that the global TAE eigenmodes are often destroyed by a large continuum damping near the plasma MHD beta limit[1,20]. Since the standard high-powered TFTR supershots were near the beta limit, it was conjectured that the TAE instability threshold could be further reduced by lowering the total plasma beta. For example, NOVA-K analysis showed that although the alpha particle drive was highest at $P=30 \mathrm{MW}$ of NBI, at that power the $n=1$ TAE modes were continuum-damped because the plasma was approaching the MHD beta limit at Ip=1.6 MA[1].

These theoretical ideas and simulations motivated the present experiment. The modeling of TAE stability for the actual Ip=2.0 MA DT discharges used in this experiment is described in Sec. 5 .

\section{Experimental Scenarios}

The goal of this experiment was to destabilize the alpha-driven TAE mode by reducing the ion temperature, while at the same time keeping the TAE drive due to the 
alpha pressure gradient as high as possible. Normally these requirements are mutually exclusive, since the DT reactivity and associated alpha pressure increase with ion temperature. Several possible "steady-state" or "transient" scenarios were considered to reconcile these requirements. One steady-state scenario was reducing the level of NBI power in a standard supershot, and another was degrading the wall conditioning at a high NBI power to form an L-mode plasma. Both of these scenarios could have reduced the ion temperature to $\mathrm{T}_{\mathrm{i}}(0) \approx 5-10 \mathrm{keV}$, but the $\mathrm{DT}$ reaction rates and alpha pressures were estimated to be a factor of $\approx 5$ below that for high fusion power supershots, and so were unacceptable.

The transient scenarios were predicated on first establishing a large alpha population, and then rapidly cooling the ions before this alpha population was thermalized. Since the expected growth time of TAE modes is relatively short $\left(<10 \mathrm{msec}\right.$ at $\mathrm{Q} / \mathrm{\Sigma}^{1} 0^{-4}$ and $f \approx 300 \mathrm{kHz}$ ), and the alpha thermalization time is normally $\approx 0.5 \sec [18]$, it was acceptable to cool the ions on the energy confinement timescale of $\approx 0.1-0.2 \mathrm{sec}$.

The simplest transient scenario is automatically obtained after the NBI is turned off, when the ion temperature and beta drop faster than the alpha pressure[21]. Early theoretical analyses had suggested that this period could be TAE unstable[1], although the analysis was uncertain due to the rapidly varying and incompletely diagnosed equilibrium and plasma profiles after NBI. This time period was examined during the initial high-powered DT discharges, but no signs of TAE instability were observed[11,13].

The scenario used for the present experiment was to transiently cool a high temperature DT supershot using He gas puffs or pellet injection during NBI. Earlier DD transport experiments had shown that high ion temperature TFTR supershots could be transformed into low ion temperature L-modes discharges within $\approx 0.1 \mathrm{sec}[22]$. It was estimated that about half the initial alpha pressure would remain after $\approx 0.1 \mathrm{sec}$, despite the larger electron density and lower electron temperature after the cooling perturbations. Therefore the alpha pressure in this transient scenario would be considerably larger than for the steady-state scenarios.

The plasma conditions used for the present experiment were very similar to the $\mathrm{Ip}=2.0 \mathrm{MA}, \mathrm{R}=2.52 \mathrm{~m}, 30 \mathrm{MW}$ NBI discharge which was initially used to obtain $6.3 \mathrm{MW}$ of fusion power (\#73268)[11]. This high temperature "baseline" DT discharge showed no signs of TAE instability, and was calculated to be stable to TAE modes by both the NOVA$\mathrm{K}$ and TAE/FL codes[2,3]. The DT discharges used in the present experiment had a slightly lower NBI power and a lower level of $\mathrm{Li}$ wall conditioning to avoid the disruptive beta limit, which was being approached in the baseline DT discharge. 


\section{Experimental Results}

There were four DT discharges in this experiment, each one having a different type of transient ion cooling perturbation: one with a small He gas puff (5.5 Torr-liters), one with a large He gas puff (18 Torr-liters), one with a single D pellet, and one with a single Li pellet. Each of these cooling perturbations began $0.6 \mathrm{sec}$ after the start of NBI, at which time the TRANSP simulations showed that the alpha particle pressure was near its maximum. The main time of interest was the $0.2 \mathrm{sec}$ just after the start of the cooling perturbation when the alpha pressure remained reasonably high.

For three of these DT discharges the maximum fusion power was in the range $\approx 5.0-5.5 \mathrm{MW}$, however, the fourth (D pellet) shot had only $\approx 3.5 \mathrm{MW}$ of fusion power (due to a NBI source fault). No severe or unusual MHD activity occurred during the times of interest, but low-level fishbone activity occurred before the cooling perturbations (as it did during the baseline DT discharge with no cooling perturbation), and sawtooth activity started $\geq 0.2 \mathrm{sec}$ after the start of the cooling perturbations. For all four DT shots there was at least one companion DD discharge, which was used to help isolate the effect of the alpha particles on the Alfven-frequency fluctuation spectra. The plasma parameters of the DD discharges were essentially the same as their companion DT discharges, except for slight beam faults. Unless otherwise noted, the data described below is for the DT discharges.

Machine and plasma parameters for these DT discharges and their DD comparison shots are shown in Table 2. All discharges had $\mathrm{Ip}=2.0 \mathrm{MA}, \mathrm{R}=2.52 \mathrm{~m}$, and $\mathrm{P} \approx 26-29$ MW of NBI power from 3-4 sec. The following sub-sections describe the plasma parameters and relevant measurements to provide sufficient information for further TAE analyses.

\subsection{Time Evolution of Plasma Parameters}

The time dependence of the line averaged electron density, the DT neutron rate, and total stored energy during these cooling perturbations are shown in Fig. 2. The NBI power was applied from 3.0-4.0 sec during these discharge, and the cooling perturbations were started at 3.6 sec. Each type of cooling perturbation had a slightly different effect on the time evolution of the plasma parameters and profiles, as expected.

The small He puff raised the line averaged density by $\approx 15 \%$ between 3.6 and 3.8 sec, while the other perturbations raised it by $\approx 50 \%$ over this time. In all cases the central electron density remained constant to within $\approx 10 \%$ during $3.6-3.8 \mathrm{sec}$, i.e. the electron density profiles were broadened from supershot-type (density peakedness $\approx 2.2$ ) 
to L-mode type (density peakedness $\approx 1.0-1.6$ ). Profiles of the electron density evolution are shown in Fig. 3.

The small He puff reduced the total plasma stored energy by $\approx 25 \%$ between 3.6 and $3.8 \mathrm{sec}$, while the other perturbations reduced it by $30-40 \%$ during this time. This is roughly the expected change from supershot toward L-mode confinement. The radial profiles of total plasma beta are also broadened toward the typical L-mode profiles shape, as shown in Fig. 4.

The time evolution of the measured ion temperatures profiles is shown in Fig. 5 for these same four DT discharges. For the three cases with full NBI power, the measured central ion temperature dropped from $T_{i}(0)=23 \pm 1 \mathrm{keV}$ to the range $T_{i}(0)=8-15 \mathrm{keV}$ within $\approx 0.2 \mathrm{sec}$, while in the fourth (lower power) discharge it dropped from $\mathrm{T}_{\mathbf{i}}(0)=19 \mathrm{keV}$ to $\mathrm{T}_{\mathrm{i}}(0)=12 \mathrm{keV}$. These decreases in central ion temperature were in the desired range for this experiment. The thermal ion beta, as shown in Fig. 6, decreased less than the thermal ion temperature due to the increased ion density. The thermal ion beta did not significantly decrease outside $r / a \approx 0.5$, however, since the increase in ion density nearly balanced the decrease in $\mathrm{T}_{\mathbf{i}}$.

The time evolution of the measured electron temperature profiles for these discharges is shown in Fig. 7. The electron temperature changed relatively little during these cooling perturbations, e.g. from $T_{e}(0)=9.0-9.5 \mathrm{keV}$ to $\mathrm{T}_{\mathrm{e}}(0)=7-8 \mathrm{keV}$ between 3.6 and $3.8 \mathrm{sec}$. Sawteeth began at $\geq 3.8 \mathrm{sec}$ in most cases, i.e. just after the time of interest (see Sec 4.5).

The alpha particle beta profile $\int_{a}(\mathrm{r})$ was calculated by the time-dependent TRANSP code using the TRANSP-calculated DT neutron rate and plasma parameters, as shown in Fig. 8. The central alpha particle beta at the start of these cooling perturbations was in the range $\int_{a}(0) \approx 0.13-0.23 \%$, which was close to the $\int_{a}(0) \approx 0.23 \%$ at the same time in the baseline DT case with $6.3 \mathrm{MW}$ of fusion power. The central alpha particle beta stayed nearly constant between 3.6 and $3.7 \mathrm{sec}$, then decreased by $\approx 20-50 \%$ between 3.7 and 3.8 sec, consistent with the decrease in the DT neutron rate and the alpha thermalization time. The shape of the $\int_{\mathfrak{a}}(\mathrm{r})$ profile stayed approximately constant during these cooling perturbations, such that the location of the peak alpha particle pressure gradient remained constant at $\mathrm{r} / \mathrm{a} \approx 0.3 \pm 0.1$.

The beam beta profiles $\int_{b}(r)$ calculated by TRANSP are shown in Fig. 9. These dropped by a larger amount, decreasing by a factor of $\approx 1.4-3$ during $3.6-3.8 \mathrm{sec}$, presumably due to the shorter beam thermalization time. Note that the beam beta was typically about 5 times higher than the alpha beta.

The $q(r)$ profiles calculated by TRANSP are shown in Fig. 10. The $q(r)$ profiles did not change significantly during the relatively short timescale of interest for this experiment, as expected. Measurements of the $\mathrm{q}(\mathrm{r})$ profile from the motional Stark effect diag- 
nostic were not available for these discharges. Measurements of $q(r)$ by MSE on similar supershots perturbed by a helium puff show no change in $q(r)$ over these timescales.

In summary, the desired scenario was obtained in each of the four DT discharges in this experiment, i.e. a transient reduction in ion temperature by about a factor of two, along with a reduction in the alpha pressure by less than a factor of two. These changes were made with modest changes in the electron density profile, with relatively little change in the electron temperature profile, and without any significant increase in background plasma MHD activity (see Sec. 4.5).

\subsection{High Frequency Magnetic Fluctuation Measurements}

Measurements of the external magnetic fluctuations were made using magnetic loops with a frequency response of up to $500 \mathrm{kHz}$ [23]. The resulting spectra of the poloidal magnetic fluctuations for the DT and comparison DD discharges are shown in Fig. 11. Each spectrum is averaged over $\pm 0.25 \mathrm{msec}$ at three times of interest, i.e. before $(3.6 \mathrm{sec})$, during $(3.7 \mathrm{sec})$, and after $(3.8 \mathrm{sec})$ the cooling perturbations.

In almost all cases there was a very small peak in the edge magnetic fluctuation spectrum at a frequency of $\approx 250-350 \mathrm{kHz}$. This peak was more than a factor of 10 times smaller than the TAE peaks seen during NBI- or ICRH-driven TAE modes in TFTR DD plasmas[13]. This peak occurred both before, during, and after the cooling perturbations in both DD and DT plasmas. Since this feature behaved very similarly for DT and DD plasmas of a given type, these peaks can not be ascribed to an alpha-driven TAE mode (the confined super-Alfvenic fusion product pressure in the DD discharges is about a factor of $\approx 50$ less than comparable DT discharges).

Since this small peak occurs in the Alfven frequency range it is being called an AFM (Alfven Frequency Mode)[24]. A systematic change in the AFM feature did occur during many of these cooling perturbations, e.g. its amplitude increased by $\mathrm{x} 2$ and its frequency decreased by $\approx 10 \%$ during the He puffs. These changes were similar for the DT and their DD comparison discharges, and seem to be correlated with the changes in edge plasma density and not to the alpha particle pressure.

\subsection{High Frequency Density Fluctuation Measurements}

Measurements were made of internal density fluctuations in the frequency range up to $500 \mathrm{kHz}$ with the beam emission spectroscopy (BES)[25] and microwave reflectometer[26]. No significant peaks in the frequency range $\geq 200 \mathrm{kHz}$ were seen for any of these discharges, in either DT or DD, implying that alpha-driven TAE modes were not present 
in this experiment, although these same diagnostics have observed NBI- and ICRH-driven TAE modes in DD experiments[7, 23].

Examples of the coherence spectra of internal density fluctuations measured by BES for these discharges are shown in Fig. 12. These spectra were taken at a major radius of $R \approx 323 \mathrm{~cm}$, corresponding to $\mathrm{q} \approx 2$ (the Shafranov shift was $\approx 15 \mathrm{~cm}$ ), and averaged over 3.6-3.9 sec. Cross-coherence spectra between two radii separated by $5 \mathrm{~cm}$ were used, which are more sensitive to coherent modes than simple auto-power spectra, since the incoherent noise in the spectra is reduced by averaging. Above $100 \mathrm{kHz}$ no peaks were observed in any of the discharges above the statistical noise represented by a coherency at the $10 \%$ level. The upper limit to possible density fluctuations, assuming a $40 \mathrm{kHz}$ bandwidth within $\approx 250-350 \mathrm{kHz}$, was $\partial \mathrm{n} / \mathrm{n} \approx 0.2-0.4 \%$. This level is much smaller than observed by BES during NBI-driven TAE experiments[27]. Below $100 \mathrm{kHz}$ there were peaks corresponding to the usual low-n coherent MHD modes in both DT and DD discharges (see Sec. 4.5).

Examples of internal density fluctuations measured by the reflectometer are shown in Fig. 13. These spectra were taken at major radii between $R=305$ and $315 \mathrm{~cm}$, corresponding $q \approx 1.5$. Again, there were no observable coherent modes in the TAE frequency range above $200 \mathrm{kHz}$, although some modes below $\approx 150 \mathrm{kHz}$ were observed in both $\mathrm{DT}$ and DD plasmas. These medium-frequency modes are described elsewhere[28].

\subsection{Alpha Particle Loss Measurements}

Measurements were made of alpha particle loss using the lost alpha scintillators[29]. The expectation was that an alpha-driven TAE mode would cause anomalous alpha particle loss, by moving passing alphas across the passing/trapped boundary to the lost alpha detector $90^{\circ}$ below the outer midplane[30]. No measurements of the confined alpha population were available for these DT discharges.

The time dependence of the alpha loss to the $90^{\circ}$ detector for the four DT discharges in this experiment is shown in Fig. 14. These signals were integrated over the pitch angle and gyroradius acceptance range of these detectors, and were normalized to the instantaneous DT neutron rate (after the neutron background was subtracted out).

In some of these DT discharges there was a gradual increase in the neutron-normalized alpha loss during the cooling perturbations, with the largest of these increases occurring during the large He puff case. This case is compared in Fig. 15 with expected first-orbit loss, which was the dominant component of alpha loss to this detector in the baseline DT discharges[31]. The measured increase of about a factor of two between 3.6$3.9 \mathrm{sec}$ agrees well with the expected increase in first-orbit loss. This increase in first-

orbit alpha loss is mainly due to the broadening of the neutron source profiles from 
supershot to L-mode, which puts a larger fraction of the alpha birth profile on unconfined loss orbits (there was little change in the calculated $q(r)$ profile during this time). There is no sharp increase in the alpha loss at the $\mathrm{Li}$ or $\mathrm{D}$ pellet injection time.

Thus there was no unexpected alpha particle loss observed during these cooling perturbations in the $90^{\circ}$ lost alpha detector. In addition, there were no signs of anomalous alpha loss by the alpha loss in the $60^{\circ}$ or $45^{\circ}$ detectors (the $20^{\circ}$ detector was not used in this experiment). There was also no time-dependent change in the pitch angle or gyroradius distributions of alpha loss during these cooling perturbations, such as previously seen during MHD-induced loss of DD fusion products[32]. Since the baseline DT discharge had a calculated global first-orbit loss of $\approx 3 \%$, the upper limit to any possible TAE-induced loss is considerably less than $3 \%$.

\subsection{Other Fluctuation Measurements}

Low frequency electron temperature and magnetic fluctuations were monitored by the electron cyclotron emission (ECE) and Mirnov diagnostics during both the DT and DD comparison discharges. An example of these signals is shown in Fig. 16 for the large He puff case in DT. The dominant fluctuations were $n=1$ fishbones at $f \approx 5 \mathrm{kHz}$, which was similar to those seen in the baseline 6.3 MW baseline discharge[13,23]. The edge magnetic perturbations from these fishbones were $>1000$ times larger than the perturbations due to the high frequency AFM mode, and generally decreased during the cooling perturbations. There were no sawteeth during the main time of interest for TAE stability between 3.6 and $3.8 \mathrm{sec}$; sawteeth began after $3.8 \mathrm{sec}$ in three of the four DT discharges in this experiment. Such sawtoothing is typical of TFTR L-mode plasmas, and was not significantly different in the DD comparison discharges.

Another fluctuation measurement was the edge ion cyclotron emission (ICE) in the frequency range up to $\approx 120 \mathrm{MHz}$. There was a significant increase in the ICE emission during the He puff perturbations, which is attributed to an alpha-driven Alfven cyclotron instability[33]. This increase in ICE is associated with an increase edge density due to the supershot-to-L-mode transition, which causes the alphas passing through the plasma edge to become super-Alfvenic. This ICE emission is apparently not related to an alpha-driven TAE mode.

\section{TAE Stability Analyses}

TAE stability analyses were made for the He puff discharges using the NOVA-K and TAE/FL codes (see Sec. 2). These analyses used the measured plasma profiles of 
$\mathrm{T}_{\mathrm{i}}(\mathrm{r}), \mathrm{T}_{\mathrm{e}}(\mathrm{r})$, and $\mathrm{n}_{\mathrm{e}}(\mathrm{r})$, along with the TRANSP analyses of derived profiles such as $\mathrm{q}(\mathrm{r})$, $\int_{a}(r)$, etc, as shown in Figs. 3-10.

Since the formalism and approximations used in these two codes are substantially different, it is not surprising that they produce somewhat different results for a given case. In general, the NOVA-K code is most useful for marginal stability due to its perturbative nature, while the TAE/FL code is an initial value code most useful for calculating unstable cases with a finite growth rate. The result was that both codes predict the TAE modes to be stable for low-n TAE modes during these cooling perturbations.

A third TAE stability code has been used to analyze other TFTR DT discharges, namely the Candy-Rosenbluth code[34]. This code predicted that the baseline DT supershot (\#73268) was weakly unstable to TAE modes with $n \leq 5$, and that electron curvature damping was the dominant damping mechanism, with thermal ion Landau damping being larger than beam ion Landau damping in this range. This code has not been used to analyze the cooling perturbations of this experiment.

\subsection{TAE Mode Structure}

The $\mathrm{n}=1-3$ TAE radial eigenmode structures as calculated by the NOVA-K code just before the cooling perturbations are shown in Fig. 17. These mode structures depend on the $n(r)$ and $q(r)$ profiles and the MHD equilibrium as derived from TRANSP. Each toroidal $n$-number has a range of $m$-numbers corresponding to various positions on the $q(r)$ profile; these different $m$-modes are coupled by toroidal effects[1-4].

A crucial result from this analysis is that the TAE modes in these plasmas were localized mainly in the outer half of the plasma, and tended to move closer to the outer edge as the $n$ mode number increased from $n=1$ to 3 . This spatial location significantly reduced the alpha drive available for the TAE instability, since the alpha particle pressure gradient was localized near $\mathrm{r} / \mathrm{a} \approx 0.3 \pm 0.1$ (see Fig. 8). The TAE eigenmodes of Fig. 17 are similar to those calculated for the 6.3 MW baseline discharge (\#73268), but different from the high-powered I=2.5 MA supershot, which had a broader pressure profile and a "core localized" TAE mode[14].

In order for alpha particles to excite a strong TAE instability, the Alfven continuous frequency spectrum should contain a "gap" across the minor radius of the plasma at a frequency near $\Sigma_{\mathrm{TAE}} \approx \mathrm{V}_{\mathrm{A}} / 2 \mathrm{qR}$. In these experiments, the TAE gap structure for the $\mathrm{n}=1$ mode remained open during both the small and large He puffs, allowing the formation of a discrete mode at $\mathrm{r} / \mathrm{a} \approx 0.6$ and $\Sigma \Sigma_{\mathrm{A}} \approx 2$, i.e. at $\mathrm{f} \approx 300 \mathrm{kHz}$. However, the $\mathrm{n}=2$ and $\mathrm{n}=3$ gap structures evolved significantly during these perturbations, as illustrated in Fig. 18 by the $n=3$ mode for the small He puff case, which "disappeared" into the Alfven continuum at $3.8 \mathrm{sec}$, i.e. a TAE mode no longer existed at this time due to continuum damping. An 
even more rapid onset of continuum damping occurred for the large He puff, according to the NOVA-K analysis.

These changes in the $n \geq 2$ TAE gap structures were an unanticipated side-effect of the density perturbations which occurred during the He puff cooling perturbations. Apparently, the increase in electron density at $r / a \approx 0.9$ caused a decrease in the local Alfven speed and a decrease of the frequency of the upper branch of the Alfven spectrum continuum. At the same time, the frequency of the lower branch of the Alfven continuum increased in the region $\mathrm{r} / \mathrm{a} \approx 0.3$, further narrowing the gap. This closure of the TAE gap structure for $n \geq 2$ inhibited the formation of a TAE mode during the time when the ion temperature was low. However, there were also other effects that contributed to the TAE stability, as discussed below.

\subsection{NOVA-K Modeling Assumptions}

The NOVA-K code $[1,2]$ is a non-variational kinetic MHD code which contains linear physics such as the ion and electron Landau damping, alpha particle drive, and beam ion damping. The effects of continuum damping and radiative damping were not included in the present calculations.

The alpha particle distribution function in NOVA-K was modeled by a classical fast ion slowing-down distribution for $\mathrm{E}_{\mathfrak{a}} \leq \mathrm{E}_{0}=3.5 \mathrm{MeV}$ :

$$
f\left(E_{a}\right) a ̊\left(E_{a}^{3} 3 / 2+E_{c}^{3 / 2}\right)^{-1}
$$

The critical energy was assumed to be the standard steady-state value of $\mathrm{E}_{\mathrm{c}} \sim 33 \mathrm{~T}_{\mathrm{e}}$. The assumed alpha energy distribution changed only through the variation of $\mathrm{E}_{\mathrm{c}}$ with $\mathrm{T}_{\mathrm{e}}(\mathrm{r}, \mathrm{t})$ during these discharges, and so did not explicitly include any non-steady state effects on the shape of the alpha distribution function such as calculated by TRANSP[35,36], e.g. due to the sudden drop of the DT reaction rate during the cooling perturbations. The Doppler broadening of about $\pm 0.5 \mathrm{MeV}$ around the alpha birth energy [37] was not included in this model.

The alpha pitch angle distribution was assumed to be isotropic, which is a fairly good approximation to the results of the Monte-Carlo simulations, at least for alphas not too near the plasma edge[35,36]. Alpha loss due to toroidal ripple effects was not included in this model for the alpha distribution, but constitutes only $\approx 10 \%$ alpha energy loss for these plasmas [38]. Finite alpha particle and beam ion orbit effects are taken into account in the NOVA-K code. The finite banana orbit size and the shift of the alphas from the magnetic flux surfaces tend to reduce the alpha particle drive at a given alpha particle pressure[17]. 
The thermal ions were specified by the measured temperature $T_{i}(r, t)$ and an ion density profile as calculated by TRANSP, with average ion mass of 2.5 for DT. There was a separate model for the neutral beam ion component, with a beam density profile based on the TRANSP analysis, and an energy distribution of the beam ion species modeled by a classical steady-state slowing-down distribution similar in form to Eq. (2), but with $\mathrm{E}_{\mathrm{c}} \sim 14 \mathrm{~T}_{\mathrm{e}}$. The beam energy component was assumed to be isotropic, which is a fairly good approximation to the time-dependent Monte Carlo beam distributions in TRANSP $[35,36]$. The relative populations and velocity distributions of co- and counter-injected $\mathrm{D}$ and $\mathrm{T}$ ions are calculated separately from TRANSP analysis for a particular discharge. Note that the separation between thermal and beam ion components was not made in the preliminary analyses of TFTR DT TAE stability (Sec. 2).

The total ion Landau damping was calculated for a given TAE n-mode by integrating the local thermal and beam ion Landau damping rate over the calculated radial eigenmode structure. Note that the thermal ion Landau damping had a different radial dependence than the beam ion Landau damping, since the thermal ion beta profile was more peaked than the beam ion beta profile (see Figs. 6 and 9).

At the beginning of the cooling perturbations the volume-averaged thermal and beam ion betas were about equal, as shown in Fig. 19, but near the location of the low-n TAE eigenmode structures at $r / a \approx 0.6$ the beam beta was higher than the thermal ion beta. The injected beam ion speed was also closer to the $\mathrm{V}_{\mathrm{A}} / 3$ resonance than the thermal ions speed (see Table 1). Therefore the beam ion damping dominated the thermal ion damping in the NOVA-K analysis.

\subsection{NOVA-K TAE Stability Results}

Time-dependent TAE stability analyses were made using the NOVA-K code for the small and large He puff cases, with the results shown in Table 3 and Fig. 20. The NOVA$\mathrm{K}$ code calculated separately each driving and damping term, as listed in Table 3 . The resulting ratio of total alpha TAE drive to total TAE damping for $n=1-3$ modes is shown in Fig. 20. The result was that all of these low-n TAE modes were predicted to be stable both before, during, and after these cooling perturbations.

The dominant damping mechanism in this analysis was the beam ion Landau damping, mainly due to the faster $\mathrm{D}$ beams rather than the slower $\mathrm{T}$ beams[14]. Since the ratio of the beam speed to the Alfven speed was close to $1 / 3$ (Table 1 ), the beam ion Landau damping also depended sensitively on the finite beam orbit size, which changed the effective parallel wavelength of the wave-particle resonance. The finite orbit effect was found to increase the beam damping by up to a factor of 5-10 with respect to the zero-orbit width case. Note that beam ion Landau damping for a slowing-down distribution such as in Eq. 
2 is much larger than a Maxwellian distribution with the same average energy, since the contribution from the slope of $f\left(E_{a}\right)$ near the cutoff energy $E_{0}$ was large.

The thermal ion Landau damping was found to be sensitive to the mode number (i.e. mode structure), but was important only for the $\mathrm{n}=1$ mode (the mode considered in Ref. 1). The thermal ion Landau damping was calculated to be relatively small, particularly for $n \geq 2$, mainly because the TAE modes in these discharges were localized in the outer half of the plasma where the ion temperature was relatively low (see Figs. 5 and 18).

After the He puffs, the calculated thermal ion Landau damping did decrease as expected, but the beam ion Landau damping and the electron Landau damping comparable to or larger than the thermal ion Landau damping, and were not significantly reduced by the He puffs (electron collisional damping was found to be negligible). After the He puff, the $n=3$ mode disappeared into the Alfven continuum, as illustrated in Fig. 18 for the small He puff case.

It is interesting that the beam ion Landau damping in the small $\mathrm{He}$ puff case actually increased between 3.6 and $3.8 \mathrm{sec}$, even though the beam ion beta decreased (Figs. 9 and 19). This was apparently due to the decrease in the Alfven speed caused by the rise in density during this time, which increases $V_{b} / V_{A}$ and so increased the beam ion damping at the $\mathrm{V}_{\mathrm{A}} / 3$ resonance.

The ratio of total alpha TAE drive to the total TAE damping increased with $\mathrm{n}$-mode number, as shown in Fig. 20 , and was $\approx 0.5$ in the least stable $(n=3)$ case. This was mainly because the electron damping and the beam ion damping were not sensitive to the $n$ mode number, whereas the alpha drive increased with the n-mode number. The conclusion from this analysis was that the experimental alpha beta was at least a factor of two below that needed to theoretically destabilize TAE mode in these discharges.

\subsection{TAE/FL Modeling Assumptions}

The TAE/FL code[3,4] is based on a gyro-Landau fluid model for Alfven frequency range instabilities. It is structured as an initial value code, which is best utilized for calculating unstable cases with finite growth rates, while NOVA-K is most useful for marginal stability due to its perturbative nature. The TAE/FL code can also be used to calculate non-linear effects, but this capability was not used for the present analyses.

In the TAE/FL analysis of these cooling perturbations the beam ions are assumed to have classical slowing-down distributions similar in form to those assumed in the NOVA$K$ code; however, the $\mathrm{D}$ and $T$ beam ions are lumped together in the TAE/FL code, whereas they are treated separately in the NOVA-K code. The alphas (since they are introduced by gyrofluid techniques) are modeled using a Maxwellian distribution whose tempera- 
ture is chosen to match a slowing-down distribution as closely as possible. The finite gyroradius of the thermal ions is taken into account, but finite orbit effects are not taken into account for either the beam ions or alpha particles. The separation between thermal and beam ion components was not made in the early analyses of TFTR DT TAE stability (Sec. 2).

This model also includes thermal ion and electron damping effects, continuum damping, and a generalized resistivity. The balance of these damping terms against the alpha drive establishes the TAE threshold. The alpha pressure threshold for TAE modes is evaluated by varying the central alpha beta (keeping the alpha profile shape constant). Individual driving and damping terms were not evaluated by this code.

\subsection{TAE/FL Code TAE Stability Results}

Time-dependent TAE stability analyses were also made using the TAE/FL code for the small and large He puff cases, with the results shown in Fig. 21. In general, the TAE mode was predicted to be stable during these cooling perturbations. However, the $n=4$ and 5 modes as analyzed by TAE/FL were much closer to TAE instability, with one case being marginally unstable ( $\mathrm{n}=4$ large $\mathrm{He}$ puff at $3.8 \mathrm{sec}$ ).

It is interesting that in the TAE/FL analysis the effect of beam ion Landau damping was not as significant as it was in the NOVA-K analysis, despite a similar model for the beam distribution function. This difference is discussed in Sec. 6.2.

\section{Discussion}

This experiment was designed to test the theoretically-predicted sensitivity of alpha-driven TAE modes to ion Landau damping. This was attempted by first creating a large alpha population in TFTR DT discharges, and then transiently reducing the ion temperature using He puff or pellet cooling perturbations.

\subsection{Summary of Experimental Results}

The intended experimental scenario was obtained with He puff and pellet cooling perturbations of DT supershots with $\approx 5 \mathrm{MW}$ of fusion power. The thermal ion temperature were successfully lowered from $T_{\mathrm{i}}(0) \approx 20 \mathrm{keV}$ to $\mathrm{T}_{\mathrm{i}}(0) \approx 10 \mathrm{keV}$ in about $0.2 \mathrm{sec}$, during which time the calculated alpha pressure was reduced by less than a factor of 2 . However, no sign of any alpha-driven TAE modes was observed during any of these cooling perturbations. These modes were expected in the frequency range $\approx 300 \mathrm{kHz}$, but were not seen by either the external magnetic coils or the internal density fluctuation mea- 
surements. In addition, no anomalous alpha particle loss was observed in the alpha loss detectors.

A small Alfven frequency mode (AFM) in the magnetic loops did increase during these cooling perturbations, but since these increases were very similar in DT and DD discharges, this mode could not have been driven by alpha particles. These AFM modes have been found to be correlated with changes in the edge plasma density[24].

\subsection{Summary of Theoretical Results}

Two independent TAE stability analyses were made based on the experimental profiles and TRANSP runs. Both predicted that the alpha-driven TAE should have been stable, despite the lowered thermal ion temperature. This is consistent with the observed absence of TAE instability in these discharges. The theoretically-predicted $\int \mathrm{a}$ threshold for TAE instability was typically a factor of 2-3 higher than the experimental value in these discharges.

A comparison of the ratio between the "experimental" alpha particle beta and the theoretically-calculated alpha beta needed for TAE instability is shown in Table 4 (the "experimental" value was derived from TRANSP analysis based on the measured profiles). Only the $n=2$ and $n=3$ cases were calculated by both codes, and in most cases the two codes agreed to within about a factor of 2-3 in this ratio. Both codes predict that the calculated TAE stability should decrease with increasing $n$-mode number, at least for $\mathrm{n} \leq 5$, mainly due to the increase in alpha drive with $\mathrm{n}$.

However, there were also some differences between the two codes, as might be expected from their different assumptions. For example, in the NOVA-K analysis the $\mathrm{n}=2$ and 3 modes moved into the Alfven continuum at 3.7 and $3.8 \mathrm{sec}$, implying that they were highly damped (NOVA-K does not calculate continuum damping explicitly). However, for the same cases the TAE/FL code did not show a substantial damping, even though it did include a model for continuum damping. This difference may be due to the sensitivity of the calculated TAE eigenmode and gap structure to the assumed profiles, as illustrated in Fig. 18.

Perhaps the largest difference between these codes concerned the effect of beam ion Landau damping. Beam ion damping dominated the total damping in the NOVA-K analysis, but was not significant in the TAE/FL analysis, as determined by turning it on and off. There are at least two possible explanations for this: (a) finite orbit effects are important (these are included in NOVA-K, but not TAE/FL), and (b) non-perturbative effects are important (these are included in TAE/FL, but not NOVA-K),

The finite beam ion orbit effect was found to be significant in the NOVA-K code, where it could increase the beam damping by a factor of 5-10. The beam ion orbit shift $\hat{\imath}$ 
is typically î/aÚ@ pol $/ R$, i.e. only about $\pm 5 \mathrm{~cm}$ for $100 \mathrm{keV}$ passing beam ions at $\mathrm{r} / \mathrm{a}=0.5$ at $\mathrm{Ip}=2.0 \mathrm{MA}$. However, the beam ion damping is very sensitive to this shift, since the beam ion speeds are near to the resonance at $\mathrm{V}_{\mathrm{A}} / 3$ (Table 1 ), and the width of this orbit shift is comparable to the TAE mode width for the most unstable $n>2$ modes. This effect was not included in the TAE/FL code.

The presence of non-perturbative effects included in the TAE/FL code imply that different damping mechanisms can interact. For example, if thermal ion Landau damping and beam ion Landau damping are suppressing the mode energy in the same ranges of poloidal mode number, then a non-perturbative calculation which takes this coupling into account is likely to show that both types of damping are less effective than in a perturbative calculation, which does not. Also, inclusion of continuum damping and nonideal effects such as ion gyroradius effects and resistivity can indirectly influence the effect of beam ion damping. These non-perturbative effects are not taken into account in the NOVA-K code.

\subsection{Implications for Future Experiments}

This experiment did not succeed in destabilizing the alpha-driven TAE mode, so further experimental work is needed in this area. Analysis of this experiment did raises several points which may be valuable for future studies of alpha-driven TAE stability.

First, these TAE stability analyses are evidently quite sensitive to small changes in the plasma profiles and equilibrium. For example, the preliminary analysis of this experiment was based on a simulated Ip=1.6 MA discharge, which was not directly relevant for the Ip=2.0 MA discharges used to obtain high fusion power in the actual experiment. Also, the theoretical treatment of beam ion Landau damping evolved during the planning of this experiment, substantially changing the results of the preliminary analysis. This implies that future DT experiments should be based on analysis of very closely analogous DD comparison discharges. For the purposes of ITER TAE studies, this also implies that a broad range of possible profiles shapes and equilibria should be examined.

Second, it seems to be absolutely necessary to have good DD comparison discharges to clearly isolate the alpha effects in DT plasmas. For example, the increases in the magnetic fluctuation spectra during the He puffs (Fig. 11) could have been mistakenly attributed to an alpha particle-driven effect if the corresponding DD comparison discharges (which also showed such an effect) were not available.

Third, a practical difficulty in these experiments was the unavoidable presence of background plasma MHD activity such as fishbones, which could have affected the TAE stability through an internal redistribution of the alpha particle pressure profile. Future experiments should try to directly measure the alpha profile in order to check the calcu- 
lated alpha particle profiles. A redistribution of alphas associated with sawteeth has recently been measured[39].

Some directions for future experiments were also brought out by the analysis of this experiment:

a) A critical factor in TAE stability was the radial location of the alpha particle pressure gradient with respect to the radial eigenmode structure. The present experiment used a standard TFTR supershot scenario in which this location was not optimal for TAE instability, i.e. the low-TAE modes were located at $r / a \approx 0.6$, whereas the alpha particle pressure gradient was maximized at $r / a \approx 0.3$. Future experiments can be better designed to align these features, either by broadening the alpha pressure profile, or by changing the TAE eigenmode structure by varying the $q(r)$ or $n(r)$ profiles[4]. Experiments on TFTR have already begun to search for TAE modes excited when $q(0)>1$, which ought to have a better alignment[40].

b) An important factor brought out by this analysis was the large effect of the beam ion Landau damping on TAE stability in TFTR. This suggests future experiments to study the post-beam phase of DT discharges when the alpha pressure remains high but the beam pressure drops significantly. So far there is no evidence for TAE instability in this time period[11-13], but more careful experiments and analysis should be done. The beam ion Landau damping might also be reduced by lowering the beam voltage or varying the species mix[14].

c) This analysis showed that the TAE instability should increase with the toroidal n-mode number. Future experiments should explore the stability of medium-n alphadriven TAE modes (i.e. $n=5-20$ ), which have already been seen in ICRH heated DD discharges[7,8] and which are also relevant to ITER[10]. Additional measurements could also look for small-scale TAE fluctuations, e.g. using microwave scattering or reflectometry focused in the appropriate $q(r)$ range.

Acknowledgments: We thank R. J. Hawryluk, H.P. Furth, D.W. Johnson, K.M. McGuire and D.M. Meade for support for this work, which was performed under DOE Contract \#DE-AC02-76CH03073. 


\section{References}

1) C.Z. Cheng, et al, Proc. 14th Int'l Conf. on Plasma Physics and Cont. Nucl. Fusion, Wurtzburg, 1992, IAEA-CN-56/D-2-1

2) C.Z. Cheng, et al, Proc. 15th Int'l Conf. on Plasma Physics and Cont. Nucl. Fusion, Seville, 1994

3) D. A. Spong, et al, Proc. 15th Int'l Conf. on Plasma Physics and Cont. Nucl. Fusion, Seville, 1994

4) D.A. Spong, et al, "Strategies for Modifying the Alpha-Driven TAE Thresholds via q-profile and Ion Temperature Control", submitted to Nucl. Fusion (1995)

5) K.L. Wong, et al, Phys. Rev. Lett. 66, 1847 (1991)

6) W.W. Heidbrink, et al, Nucl. Fusion 31, 1635 (1991)

7) R.Wilson, et al, Proc. 14th Int'l Conf. on Plasma Physics and Cont. Nucl. Fusion, IAEA-CN-56/E-2-2, p. 661, Wurtzburg, 1992

8) M. Saigusa, et al, Plasma Phys. Control. Fusion 37, 295 (1995)

9) R.J. Hawryluk, et al, Proc. 15th Int'l Conf. on Plasma Physics and Cont. Nucl. Fusion, Seville, 1994

10) S. Putvinski, et al, Proc. 15th Int'l Conf. on Plasma Physics and Cont. Nucl. Fusion, Seville, 1994

11) J. D. Strachan, et al, Phys. Rev. Lett. 72, 3526 (1994)

12) K.M. McGuire, et al, Physics of Plasmas 2(6) 2176 (1995)

13) E.D. Fredrickson, et al, Proc. 15th Int'l Conf. on Plasma Physics and Cont. Nucl. Fusion, Seville, 1994

14) G.Y. Fu, et al, submitted Phys Rev. Letters, 1995

15) G.Y. Fu and J. W. Van Dam, Phys. Fluids B1, 1949 (1989)

16) H.P. Furth, et al, Nucl. Fus. 30, 1799 (1990)

17) G.Y Fu and C.Z. Cheng, Phys. Fluids B 4, 3722 (1992)

18) R. V. Budny, Nucl. Fus. 32,429 (1992)

19) D.A. Spong, et al Proc. IEA Workshop in Alpha Physics and Tritium Issues in Large Tokamaks, PPPL, Feb. 1993

20) H. Biglari and L. Chen, Phys. Rev. Lett. 67, 3681 (1991)

21) S.J. Zweben, et al, Nucl. Fusion 28, 2230 (1988)

22) S. D. Scott, et al, 19th EPS Conference, Kiev, p. I-39 (1992)

23) E.D. Fredrickson, et al, Proc. EPS Conf., Montpellier (1994) p. 246

24) Z. Chang, et al, submitted to Nucl. Fusion, 1995

25) S. Paul and R.J. Fonck, Rev. Sci. Inst. 61, 3498 (1990)

26) E. Mazzucato, R. Nazikian, S. Scott, et al, Proc. 22nd EPS Conference, Bornemouth, UK, 1995 
27) R. Durst, et al, Phys. Fluids B 4, 3707 (1992)

28) R. Nazikian, et al, to be submitted to Physics of Plasmas

29) D.S. Darrow, et al Rev. Sci. Inst. 63, 476 (1995)

30) D.J. Sigmar and C.T. Hsu, Phys. Fluids B 4, 1506 (1992)

31) S.J. Zweben, et al, to be published in Nucl. Fusion, 1995

32) S.J. Zweben, et al Physics of Plasmas 1, 1994, 1469

33) S. Cauffman, et al, submitted to Nucl. Fusion (1995)

34) J. Candy and M.N. Rosenbluth, to be published in Nucl. Fusion 1995

35) R. V. Budny, Nucl. Fus. 34, 1247 (1994)

36) R.V. Budny, et al, submitted to Nuclear Fusion, 1995

37) G. Sadler and P. Van Belle, Proc. 22nd EPS Conference, Bornemouth, UK, 1995

38) M.H. Redi, et al, Proc. 22nd EPS Conference, Bornemouth, UK, 1995

39) M. Petrov, et al, submitted to Nucl. Fusion, 1995

40) S. Batha, et al, submitted to Nucl. Fusion, 1995

\section{Figure Captions}

1) Simplified theoretical dependence of the thermal ion Landau damping $\oplus_{i} / \Sigma_{0}$ (normalized to the TAE frequency) on the ion beta. Ion Landau damping is expected to be very sensitive to the ion beta over the range of this experiment, as indicated at the bottom of this figure.

2) Time dependence of line averaged density, DT neutron rate, and total plasma stored energy for the four DT discharges in this experiment. The cooling perturbations begin at $3.6 \mathrm{sec}$ in all cases. These perturbations change a supershot plasma to an L-mode plasma in $\approx 0.1$ sec. The time dependences of the companion DD plasmas are very similar.

3) Evolution of the electron density profiles for the DT discharges in this experiment, as measured by the multichannel interferometer. The density profiles broaden during these cooling perturbations, but the central densities are approximately unchanged.

4) Evolution of the total toroidal $\int$ profiles for the DT discharges in this experiment, as. calculated by TRANSP. The central $\int$ decrease significantly, but the $\int$ in the outer half of the plasma remain approximately unchanged.

5) Evolution of the ion temperature profiles for the DT discharges in this experiment, as measured by the CHERS diagnostic. The central ion temperatures fall from $\mathrm{T}_{\mathrm{i}}(0) \approx 19-23$ $\mathrm{keV}$ to $\mathrm{T}_{\mathrm{i}}(0) \approx 8-15 \mathrm{keV}$ during these discharges within $0.2 \mathrm{sec}$ after the start of the cooling perturbations. 
6) Evolution of the thermal ion beta profiles for the DT discharges in this experiment, as calculated by TRANSP. The central thermal ion beta falls by $\approx 50-250 \%$ within $\approx 0.2 \mathrm{sec}$ after these cooling perturbations. However, the thermal ion betas at $\mathrm{r} / \mathrm{a} \geq 0.5$ do not change significantly during this time, due to the rise in density associated with these cooling perturbations.

7) Evolution of the measured electron temperature profiles for the DT discharges in this experiment, as measured by the ECE diagnostic. The temperature profiles are relatively unaffected during the $\approx 0.2 \mathrm{sec}$ after the start of these cooling perturbations.

8) Evolution of the calculated alpha beta profiles the DT discharges in this experiment, as calculated by TRANSP. These calculations assume classical alpha particle confinement and thermalization (the $\approx 10 \%$ additional alpha energy loss due to TF ripple loss is not included). The alpha particle betas fall by less than a factor of two during the $\approx 0.2 \mathrm{sec}$ after the start of these cooling perturbations.

9) Evolution of the beam beta profiles for the DT discharges in this experiment, as calculated by TRANSP. During the cooling perturbations the beam betas are reduced more than the alpha particle betas, presumably due to the shorter beam thermalization time. These profiles include both the $\mathrm{D}$ and $\mathrm{T}$ beam components.

10) Evolution of the $q(r)$ profiles as calculated by TRANSP for the four DT discharges in this experiment. The $q(r)$ profiles do not change appreciably during the $0.2 \mathrm{sec}$ after the start of the cooling perturbations, due to the long resistive relaxation time in these high temperature plasmas. The $q(r)$ profiles were not measured during this experiment.

11) Magnetic fluctuation spectra measured for DT and DD comparison discharges for: (a) the He puff perturbations, and (b) the pellet perturbations. The dashed lines are for a time just before the cooling perturbations $(3.6 \mathrm{sec})$, the solid lines are for a time during the cooling perturbations $(3.7 \mathrm{sec})$, and the gray lines are after the cooling perturbations $(3.8 \mathrm{sec})$. These spectra were taken from a coil located $67^{\circ}$ above the outer midplane. In all cases there is a small peak in the Alfven range of frequencies near $300 \mathrm{kHz}$, but since the behavior of this peak is very similar between DT and DD discharges, it could not have been due to an alpha-driven TAE mode. The changes in this Alfven frequency mode (AFM) correlate with changes in the edge plasma density. 
12) Examples of BES coherency spectra during the cooling perturbations for the small He puff case (top) and the Li pellet case (bottom). There were no measurable coherent density fluctuations above $100 \mathrm{kHz}$ in any of these DT or DD discharges. These crosspower spectra were centered at $R=323 \mathrm{~cm}(q \approx 2)$ with a radial separation of $5.5 \mathrm{~cm}$, and averaged over 3.6-3.9 sec.

13) Density fluctuation spectra from the reflectometer spectra for: (a) the He puff perturbations, and (b) the pellet perturbations, taken between $305 \mathrm{~cm}$ and $315 \mathrm{~cm}$ major radius $(\mathrm{q} \approx 1.5)$. The reflectometer phase spectra are shown for DT and companion DD discharges. No indication of TAE mode activity in the Alfven range of frequencies was observed before or after the cooling perturbations in either the DD or DT plasmas.

14) Neutron-normalized alpha particle loss signals measured by the lost alpha scintillator detector $90^{\circ}$ below the outer midplane. In the large He puff and Li pellet cases, the alpha loss increased slowly during the cooling perturbation. However, this increase was due to the broadening of the alpha source profile, and not an excitation of alpha-driven TAE modes. A similar absence of alpha loss due to alpha-driven TAE modes is also seen on the other lost alpha detectors.

15) A comparison of neutron-normalized alpha loss in the $90^{\circ}$ detector with the calculated first-orbit loss for the large He puff case. The increase observed between 3.6 and 3.9 sec is explained by the broadening of the neutron source profile during the transition from a supershot to an L-mode, and does not imply any alpha loss due to an alpha-driven TAE mode. The uncertainties in the data and modeling are each $\approx 10-20 \%$ at all points.

16) Low frequency MHD activity during the large He puff case, as measured by the electron cyclotron emission (ECE) temperature diagnostic and the Mirnov magnetic pickup loop. This and all other discharges in this experiment had $\approx 5 \mathrm{kHz}$ fishbone oscillations during NBI, which generally decreased in amplitude during the cooling perturbations. This MHD activity did not cause a measurable alpha particle loss, but may have caused an internal rearrangement of the alpha density profile (not measured in this experiment). Sawteeth appear only after $3.8 \mathrm{sec}$ in all of these discharges.

17) TAE radial eigenmode structures for $n=1-3$ just prior to the cooling perturbation for the small He puff case, as calculated by the NOVA-K code (i.e. \#75926 at $3.6 \mathrm{sec}$ ). The vertical axis in these plots is the relative displacement of the magnetic flux surfaces due to these eigenmodes for each $\mathrm{m}$-component at a given $\mathrm{n}$ (the horizontal axis is normalized to the minor radius). The TAE mode structures are mainly located in the outer half of the 
minor radius in these discharges, which is not optimal for coupling to the alpha particle pressure gradient, which is located near $\mathrm{r} / \mathrm{a} \approx 0.3$.

18) TAE Alfven frequency spectra at different times for the $\mathrm{n}=3$ mode for the small $\mathrm{He}$ puff case (the frequencies are normalized to the Alfven frequency, and the horizontal axes are normalized to the minor radius). The TAE gap mode exists just below the upper branch of the Alfven continuum at 3.6 and $3.7 \mathrm{sec}$, but moves into the Alfven continuum and disappears at $3.8 \mathrm{sec}$ due to the change in density profile caused by the He puff. The TAE frequency of $\left(\Sigma / \Sigma_{T A E}\right)^{2} \approx 5-6$ corresponds to $\mathrm{f} \approx 300 \mathrm{kHz}$.

19) Volume-averaged toroidal betas vs. time for the small He puff case, as calculated by TRANSP. The volume-averaged beam beta and thermal ion beta are comparable just before the cooling perturbation, but the beam beta decreases more than the thermal beta after the cooling perturbations. However, the beam ion beta is larger than the thermal ion beta at the location of the TAE resonances at r/a $\geq 0.5$ (see Fig. 6 and 9 ).

20) Ratio of the alpha particle TAE drive divided by the total TAE damping for $n=1-3$ modes, as calculated by NOVA-K for the small and large He puff cases. This ratio is $\leq 1$ for all cases, implying TAE modes should be stable, which is consistent with the experimental results.. The TAE modes are slightly less stable just after the cooling perturbations at $3.7 \mathrm{sec}$, but the alpha drive is still a factor of two lower than needed for TAE instability.

21) Theoretically predicted alpha-driven TAE thresholds vs. time for the small and large He puff cases as calculated by the TAE/FL code, along with the experimental alpha betas calculated for these cases by TRANSP. The alpha pressure profile is assumed to remain constant vs. time as the central alpha particle beta varies. For all cases these discharges are predicted to be TAE stable, except perhaps for the $n=4$ case at 3.8 sec during the large He puff, when the stability is marginal. 
Table 1 - TAE Stability Parameters for DT Baseline Case

(TRANSP analysis of \#73268 at 3.8 sec, no cooling perturbation)

\begin{tabular}{|c|c|}
\hline $\mathrm{V} \alpha 0=1.3 \times 10^{9} \mathrm{~cm} / \mathrm{sec}$ & DT alpha birth speed $\quad(\mathrm{E}=3.5 \mathrm{MeV})$ \\
\hline $\mathrm{V}_{\mathrm{A}}(0)=7 \times 10^{8} \mathrm{~cm} / \mathrm{sec}$ & Central Alfven speed $\quad\left(n_{e}=7 \times 10^{13} \mathrm{~cm}^{-3}, B=5\right.$ T, DT $)$ \\
\hline $\mathrm{V}_{\alpha 0} / \mathrm{V}_{\mathrm{A}}(0)=1.8$ & Alpha birth speed/Central Alfven speed \\
\hline$V_{i}(0) / V_{A}(0)=0.15$ & $\begin{array}{l}\text { Thermal ion speed/Central Alfven speed } \\
\qquad\left(\mathrm{T}_{\mathrm{i}}(0)=25 \mathrm{keV}, \mathrm{M}=2.5\right)\end{array}$ \\
\hline $\mathrm{V}_{\mathrm{bD}} / \mathrm{V}_{\mathrm{A}}(0)=0.45$ & $\begin{array}{l}\text { Deuterium neutral beam speed/Central Alfven } \\
\text { speed }\left(E_{b}=100 \mathrm{keV}\right)\end{array}$ \\
\hline $\mathrm{V}_{\mathrm{bT}} / \mathrm{V}_{\mathrm{A}}(0)=0.37$ & $\begin{array}{l}\text { Tritium neutral beam speed/Central Alfven } \\
\text { speed }\left(E_{b}=100 \mathrm{keV}\right)\end{array}$ \\
\hline$\beta(0)=3.6 \%$ & Total plasma beta on axis \\
\hline$\beta_{\alpha}(0)=0.22 \%$ & Alpha beta on axis \\
\hline$\beta_{\mathrm{i}}(0)=1.8 \%$ & Thermal ion beta on axis \\
\hline$\dot{\beta}_{b}(0)=0.35 \%$ & Total beam ion beta on axis \\
\hline
\end{tabular}




\section{Table 2 - Shot List}

All shots at I=2.0 MA, R=2.52 $\mathrm{m}$ (DT shots in bold)

$\begin{array}{llccc}\text { shot \# } & \begin{array}{l}\text { Shot } \\ \text { Type }\end{array} & \begin{array}{l}\text { NBI Power } \\ \text { MW @ 3.6 sec }\end{array} & \begin{array}{l}\text { Ti(0) } \\ \text { @ } \\ 3.8 \mathrm{sec}\end{array} & \begin{array}{c}\text { Peak Neutron rate } \\ (\mathrm{x} 1018 / \mathrm{sec})\end{array} \\ 75923 & \text { sm He DD } & 26 & & \\ 75926 & \text { sm He DT } & 28 & 15 \mathrm{keV} & <0.03 \\ 75930 & \text { lg He DD } & 25 & & 1.8 \\ 75932 & \text { lg He DT } & 29 & 8 \mathrm{keV} & <0.05 \\ 75934 & \text { D pel DD } & 27 & & 1.8 \\ 75936 & \text { D pel DT } & 27 & 12 \mathrm{keV} & <0.07 \\ 75938 & \text { Li pel DD } & 27 & 11 \mathrm{keV} & <0.07 \\ 75941 & \text { Li pel DT } & 29 & & 1.9 \\ 73268 & \text { baseline } & 30 & 25 \mathrm{keV} & 2.2 \\ 75919 & \text { baseline } & 27 & & <0.03 \\ 75922 & \text { baseline } & 27 & & <0.03\end{array}$




\section{Table 3 - TAE Stability Analysis (Nova-K)}

\section{Small He puff (\#75926)}

$\underline{\mathrm{n}}=1$ mode

Time $\gamma \mathrm{ILD}$

3.6

3.7

$0.14 \% \quad 0.14 \%$

$0.15 \% \quad 0.14 \%$

3.8

$0.02 \% \quad 0.15 \%$ భbeam

$0.69 \%$

$0.97 \%$

$1.16 \%$ yo $\gamma \alpha / \gamma d a m p$

$0.06 \% \quad 0.05$

$0.08 \% \quad 0.06$

$0.05 \% \quad 0.04$
Large He puff (\#75932)

\section{$\underline{n}=1$ mode}

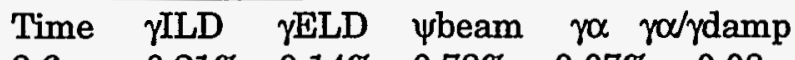

$\begin{array}{llllll}3.6 & 0.21 \% & 0.14 \% & 0.78 \% & 0.07 \% & 0.08\end{array}$

$\begin{array}{lllllll}3.7 & 0.11 \% & 0.13 \% & 1.6 \% & 0.10 \% & 0.06\end{array}$

$\begin{array}{llllll}3.8 & 0.1 \mathrm{e}-4 & 0.16 \% & 0.86 \% & 0.06 \% & 0.06\end{array}$ $\underline{n}=2$ mode

Time $\gamma$ ILD

3.6

3.7

$0.03 \%$

$0.02 \%$

$0.01 \% \quad 0.16 \%$

3.8
Ybeam

$0.27 \%$

$0.31 \%$

$0.38 \%$ $\gamma \alpha \quad \gamma \alpha / \gamma$ damp

$0.14 \% \quad 0.31$

$0.16 \% \quad 0.34$

$0.10 \% \quad 0.19$ $\mathrm{n}=2$ mode

Time $\gamma$ ILD $\gamma E L D$ beam $\gamma \alpha \quad \gamma \alpha / \gamma$ damp

$\begin{array}{llllll}3.6 & 0.05 \% & 0.14 \% & 0.30 \% & 0.14 \% & 0.32\end{array}$

$\begin{array}{lllllll}3.7 & 0.02 \% & 0.14 \% & 0.69 \% & 0.18 \% & 0.22\end{array}$

$3.8>$ the mode goes in continuum.

$\mathrm{n}=3$ mode

$\underline{\mathrm{n}=3 \text { mode }}$

Time $\gamma I L D \quad \gamma E L D \quad \psi b e a m \quad \gamma \alpha \quad \gamma \alpha / \gamma$ damp

$\begin{array}{llllll}3.6 & 0.02 \% & 0.14 \% & 0.30 \% & 0.19 \% & 0.41\end{array}$

$\begin{array}{llllll}3.7 & 0.02 \% & 0.13 \% & 0.30 \% & 0.22 \% & 0.49\end{array}$

3.8 > the mode goes into continuum.

Time $\gamma I L D$ yELD $\psi$ beam $\gamma \alpha \quad \gamma \alpha / \gamma$ damp

$\begin{array}{llllll}3.6 & 0.08 \% & 0.13 \% & 0.39 \% & 0.20 \% & 0.50\end{array}$

3.7

3.8 > the mode goes in continuum.

yILD = ion Thermal Landau damping

$\gamma E L D=$ electron Landau damping

భbeam

$\gamma \alpha$

= beam ion Landau damping

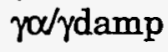

$=$ alpha drive

= alpha drive/total damping 
Table 4 - Comparison of Calculated TAE Stability Thresholds

experimental $\beta \alpha$ (TRANSP)

theoretical $\beta \alpha$ for TAE mode

\begin{tabular}{lcccc} 
Case & Time & n & NOVA-K & TAE/FL \\
\hline sm He & 3.6 & 2 & 0.31 & 0.12 \\
sm He & 3.6 & 3 & 0.41 & 0.12 \\
& & & & \\
lg He & 3.6 & 2 & 0.32 & 0.37 \\
lg He & 3.6 & 3 & 0.50 & 0.37 \\
& & & & \\
sm He & 3.7 & 2 & 0.34 & 0.16 \\
sm He & 3.7 & 3 & 0.41 & 0.21 \\
& & & & \\
$\operatorname{lg~He}$ & 3.7 & 2 & 0.22 & 0.63 \\
$\operatorname{lg~He}$ & 3.7 & 3 & cont & 0.63 \\
& & & & \\
sm He & 3.8 & 2 & 0.19 & 0.09 \\
sm He & 3.8 & 3 & cont & 0.20 \\
& & & & \\
$\operatorname{lg~He}$ & 3.8 & 2 & cont & 0.38 \\
$\lg \mathrm{He}$ & 3.8 & 3 & cont & 0.38
\end{tabular}




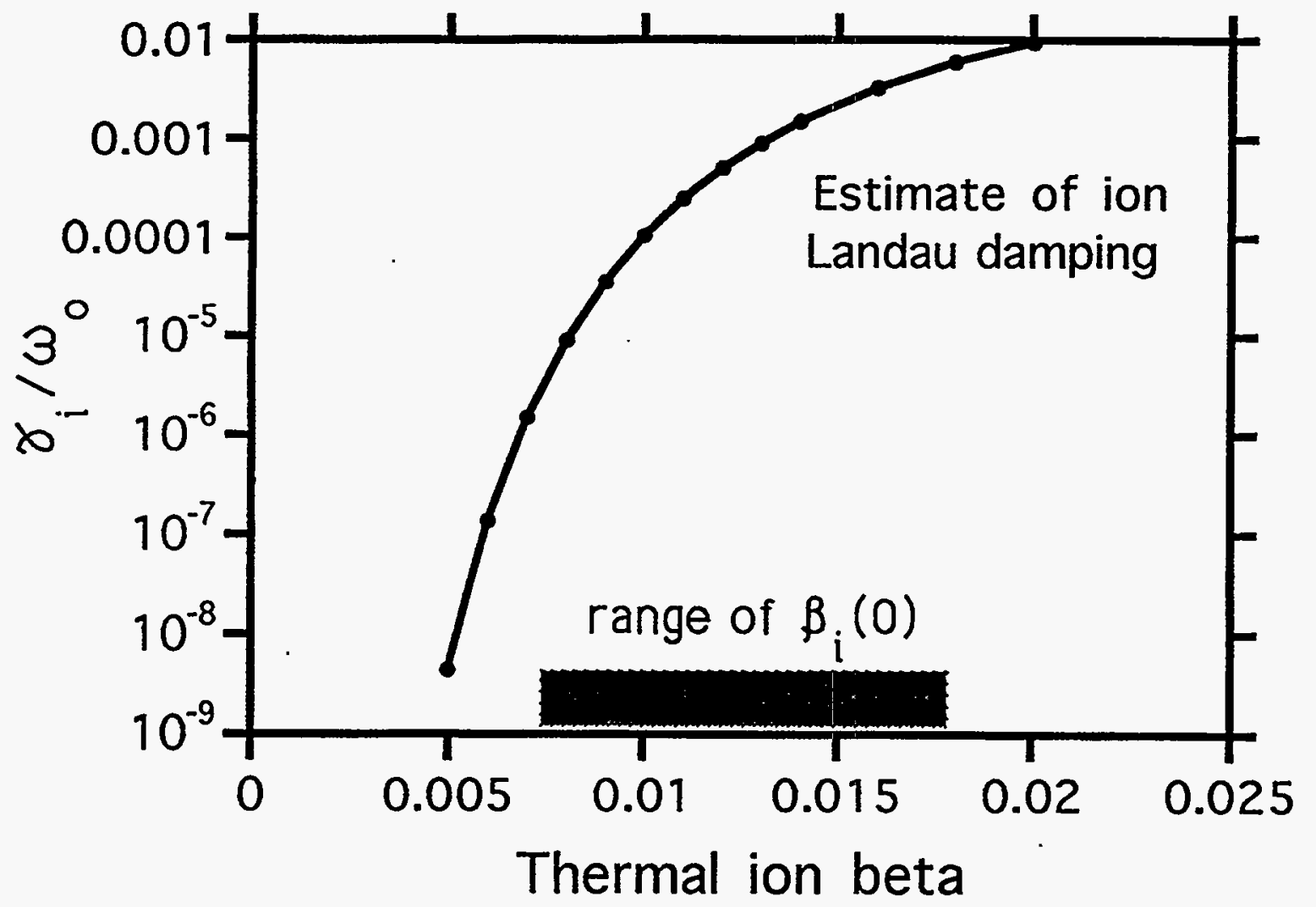

Fig. 1 

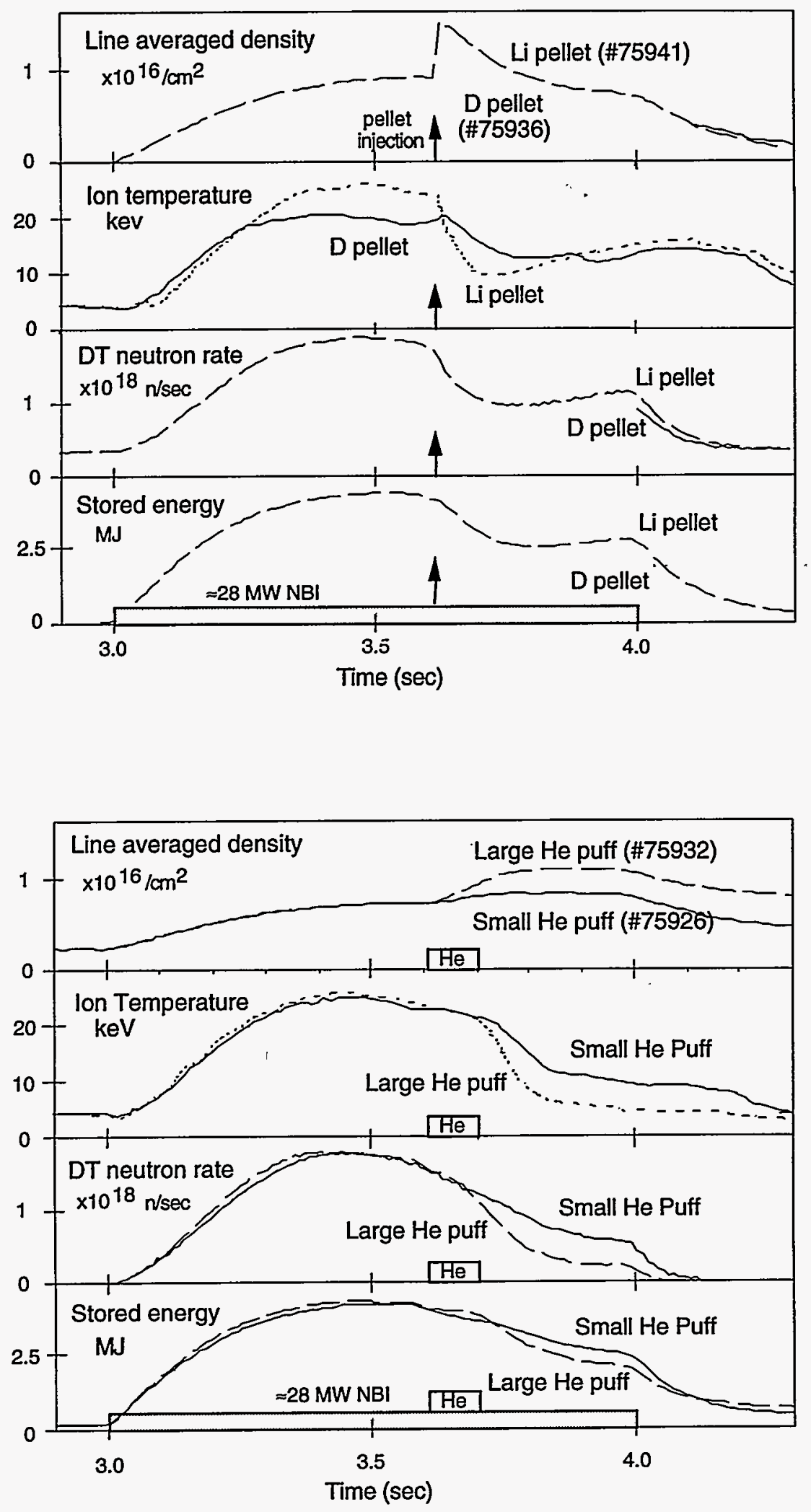

Fig. 2 


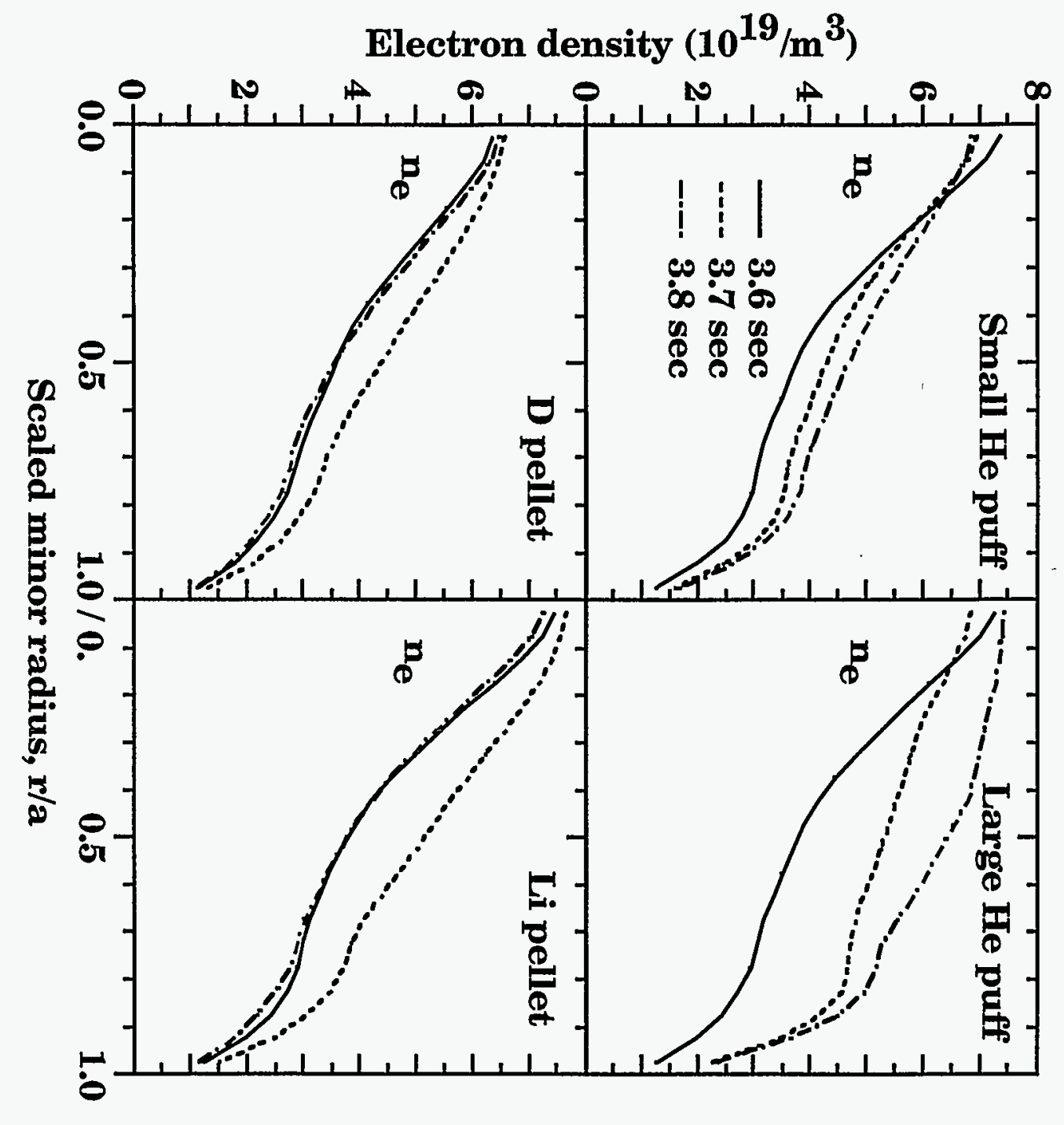




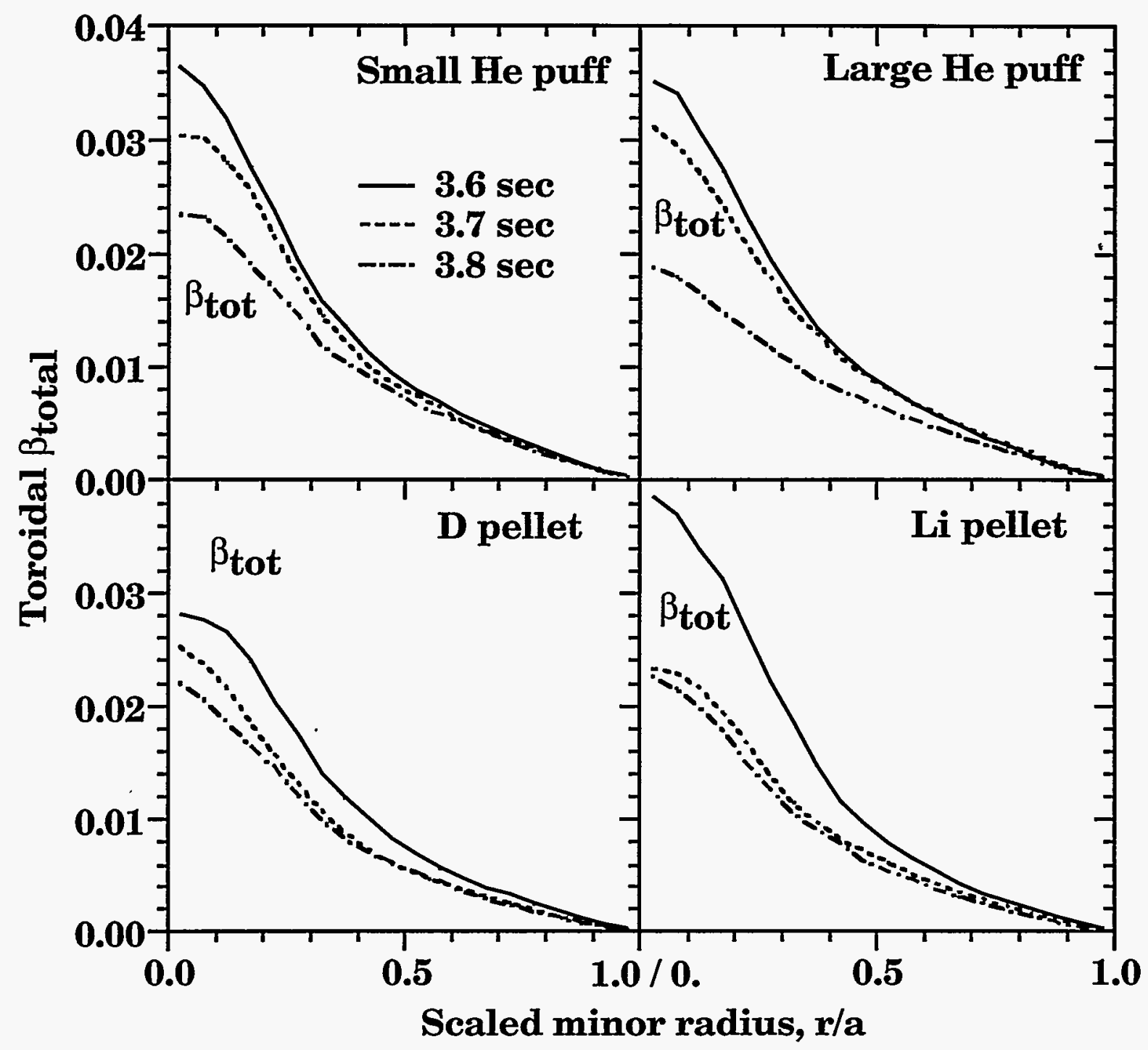

Fig. 4 


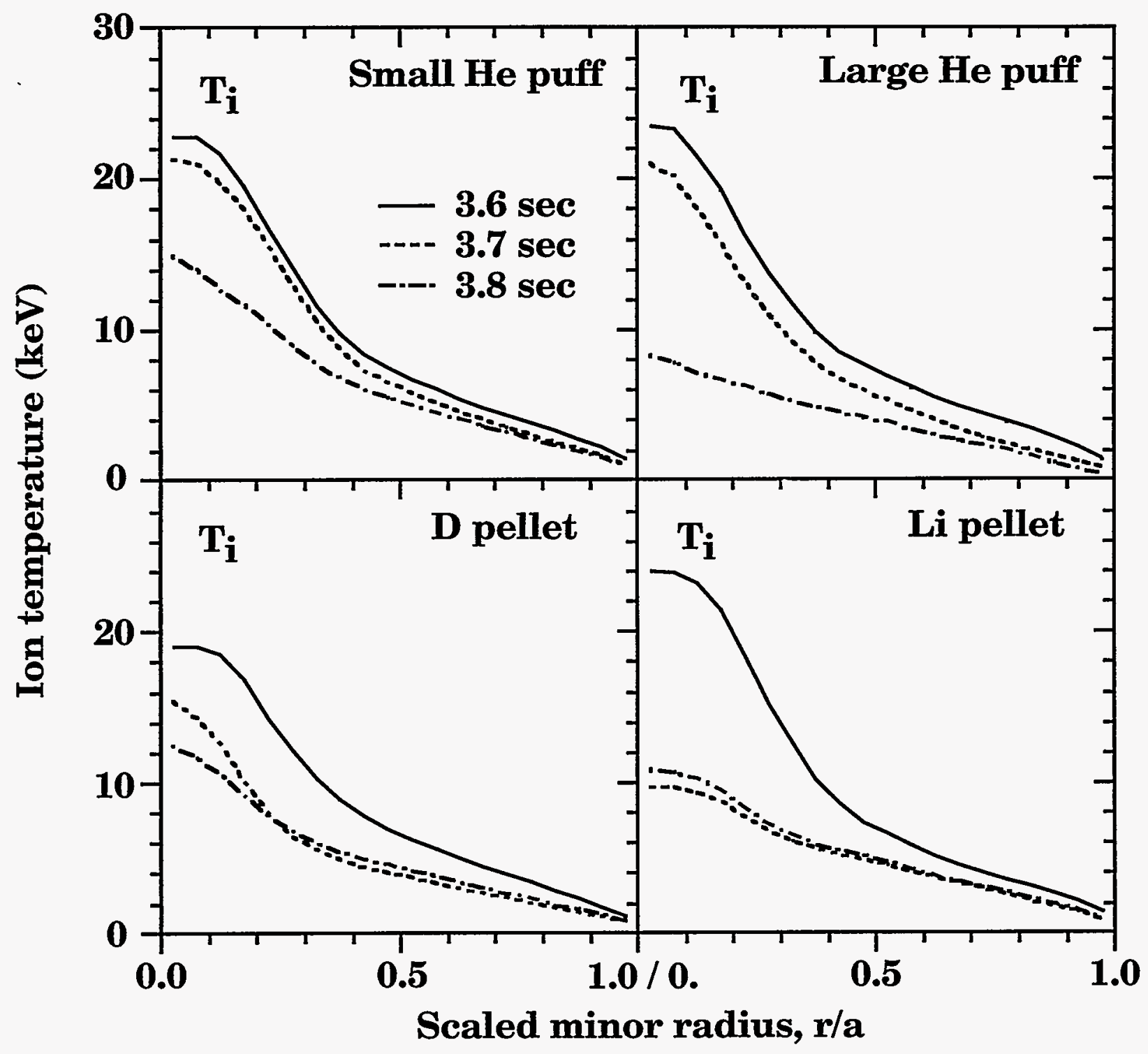

Fig. 5 


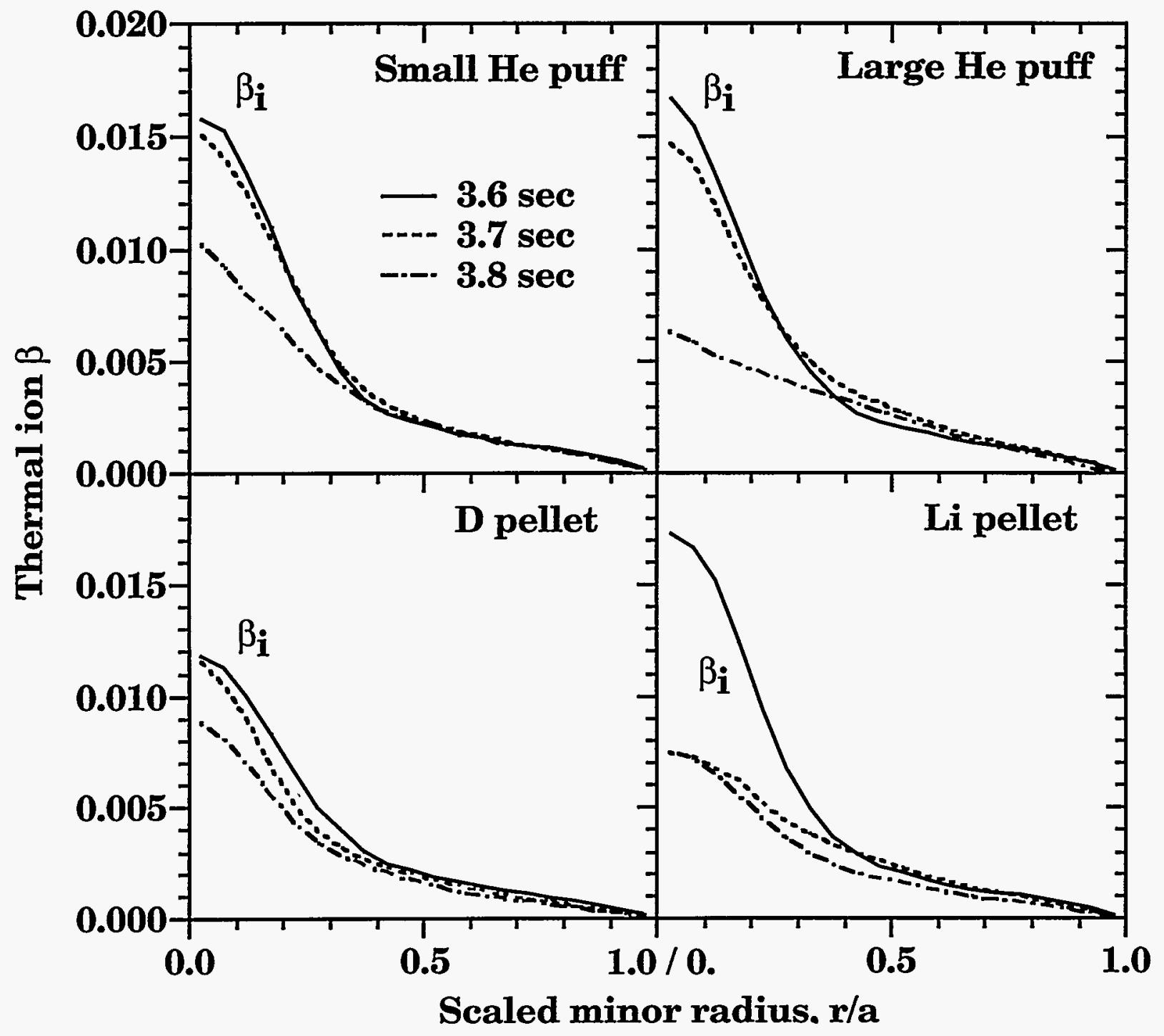

Fig. 6 


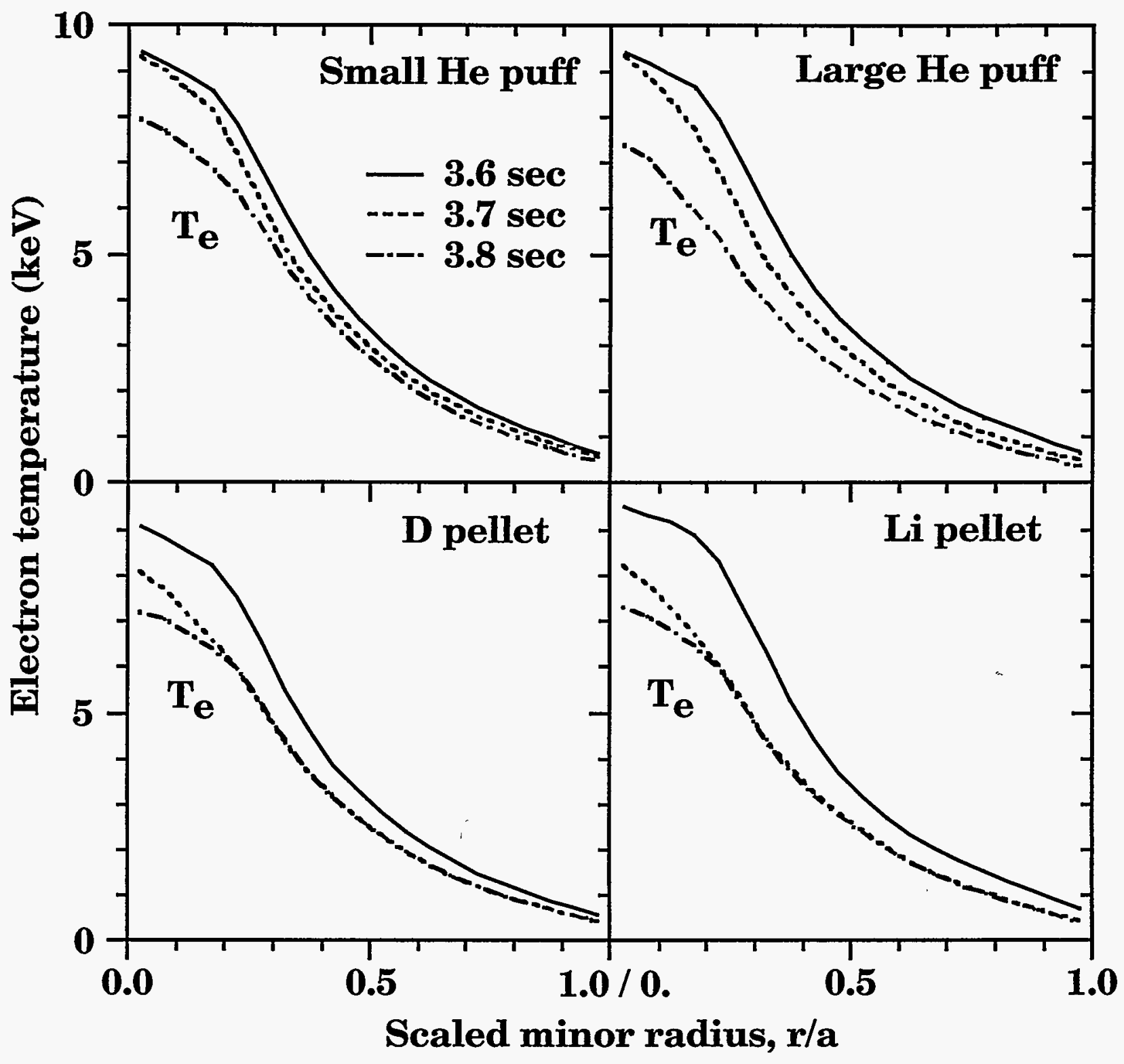

Fig. 7 


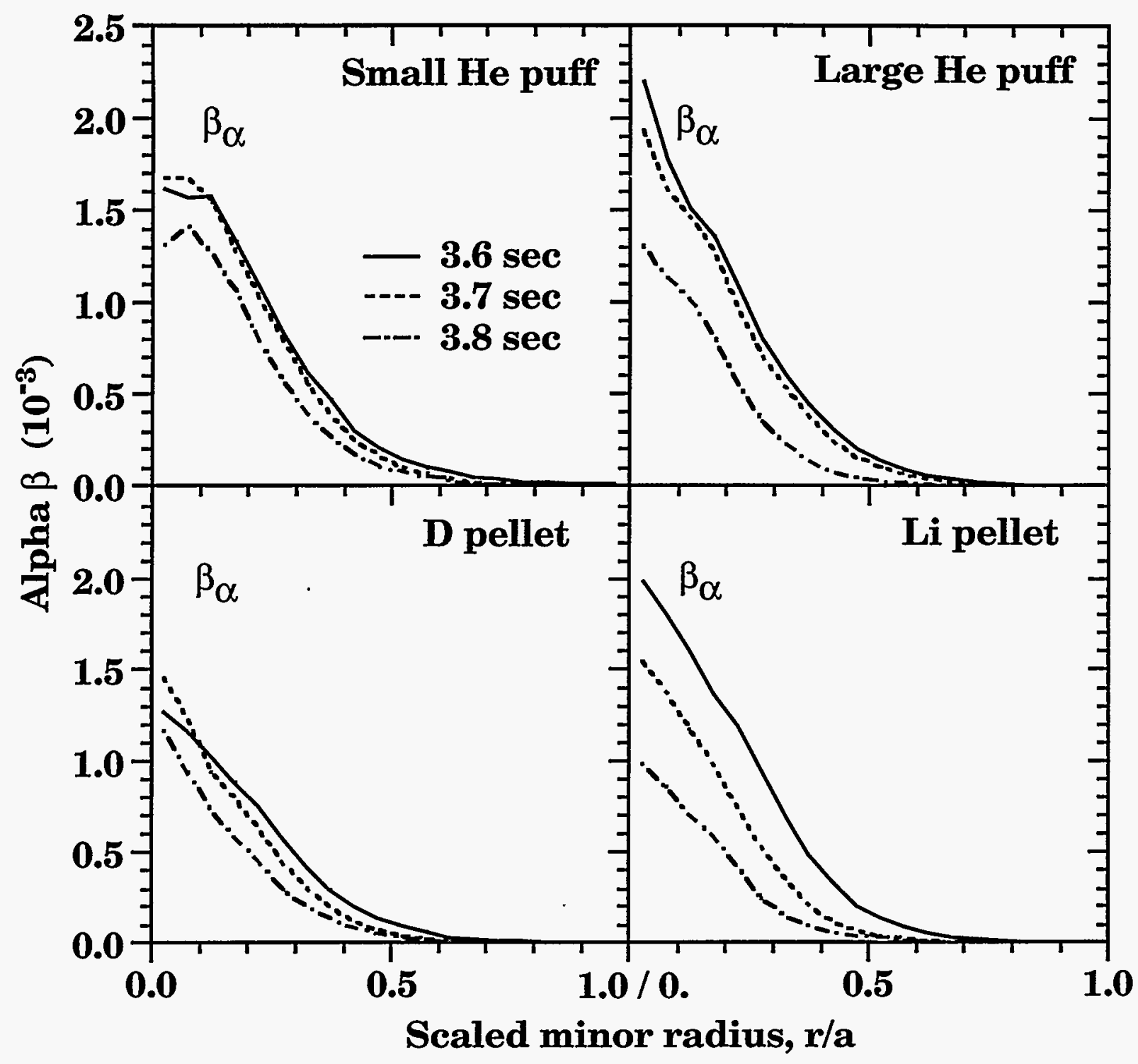

Fig. 8 


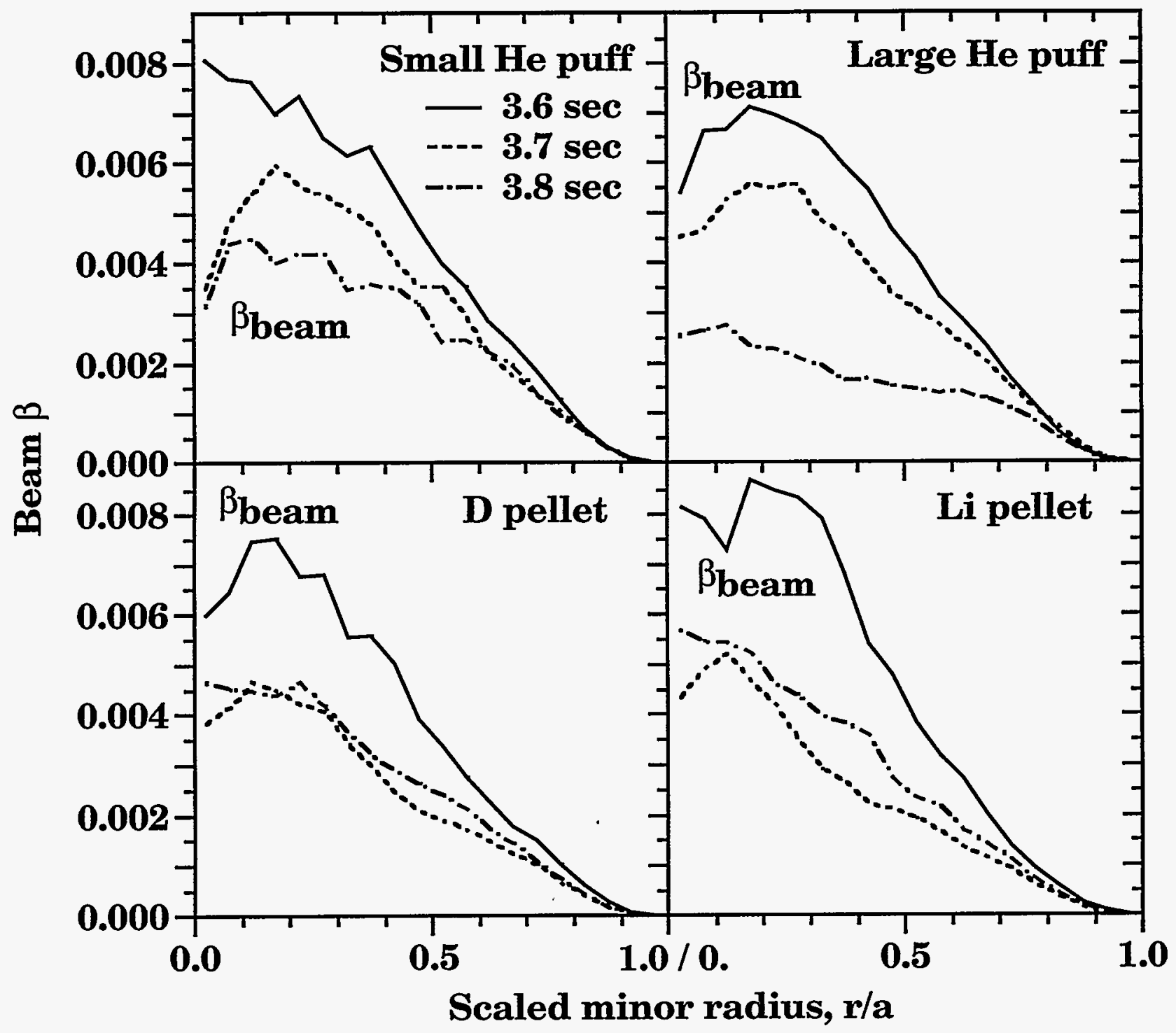

Fig. 9 


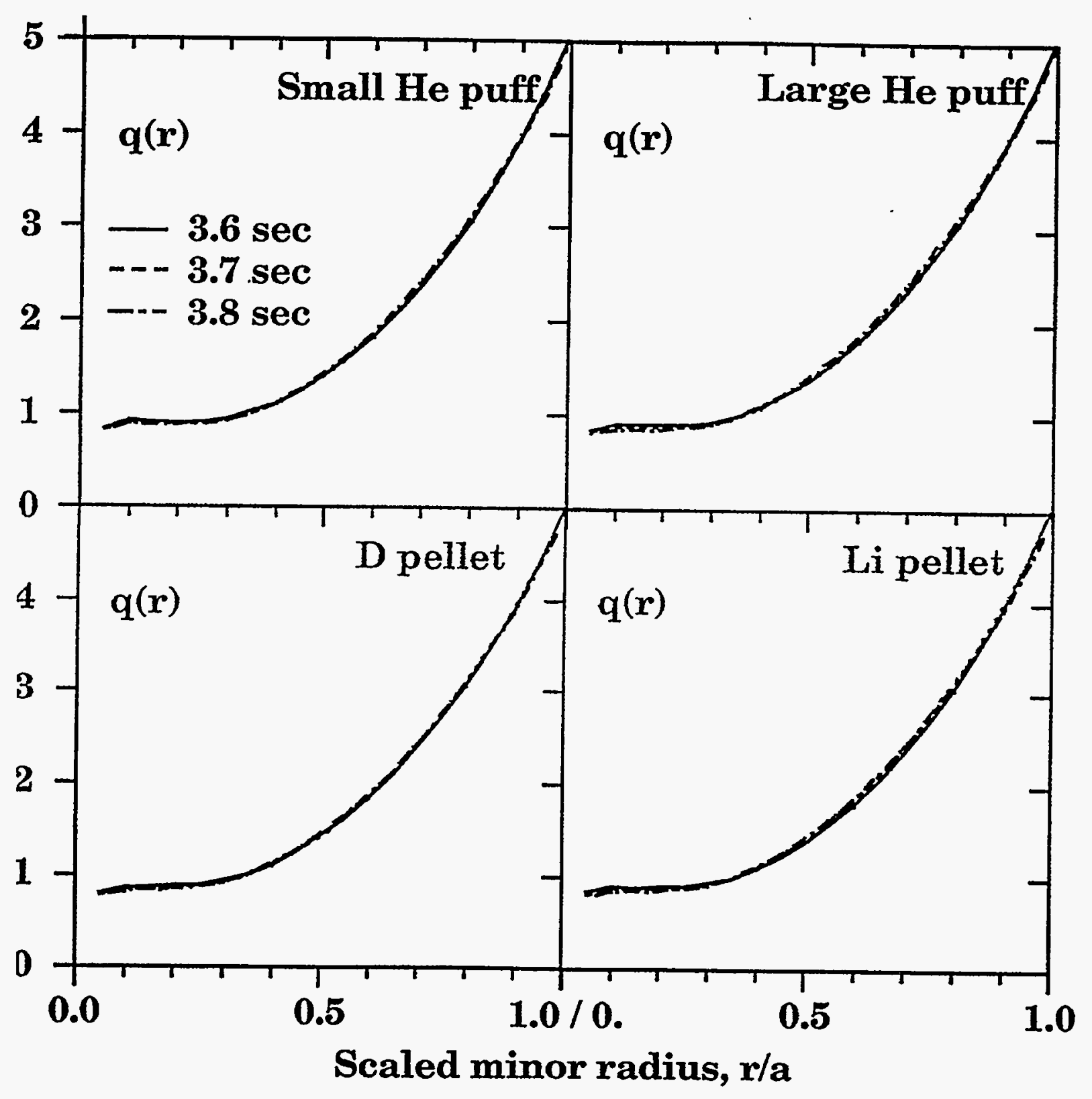

Fig. 10 

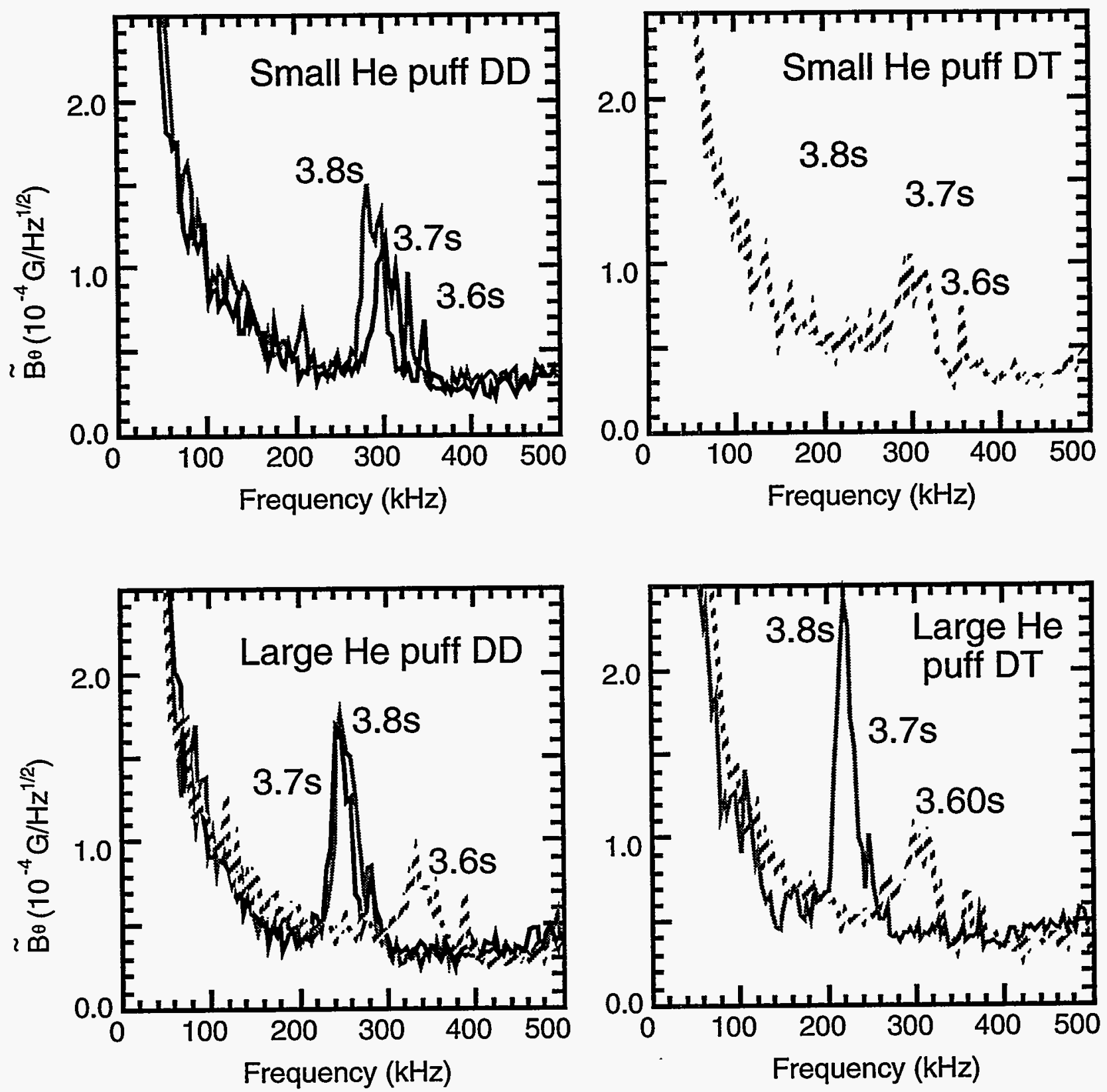

Fig. 11(a) 

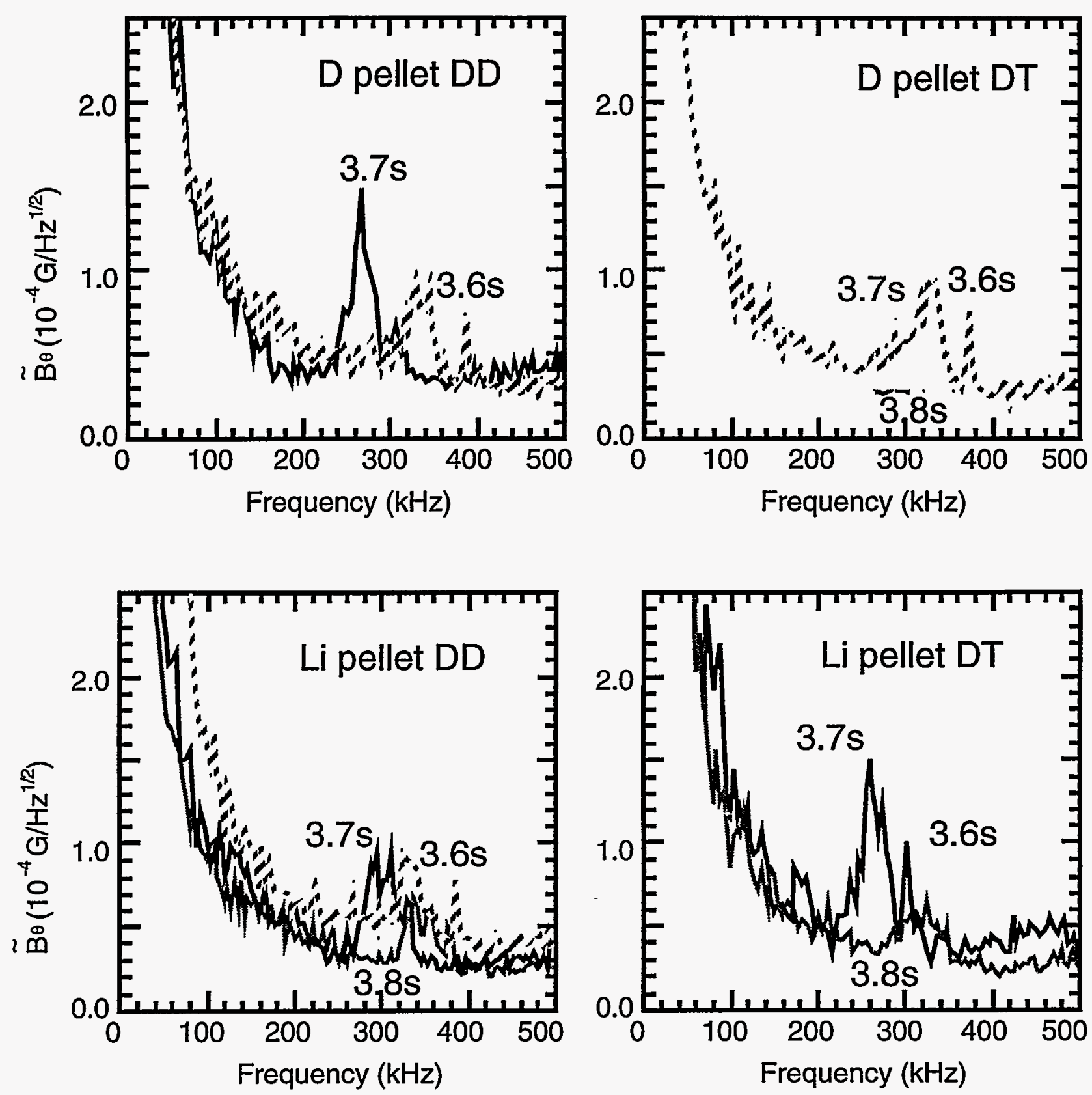

Fig. 11(b) 

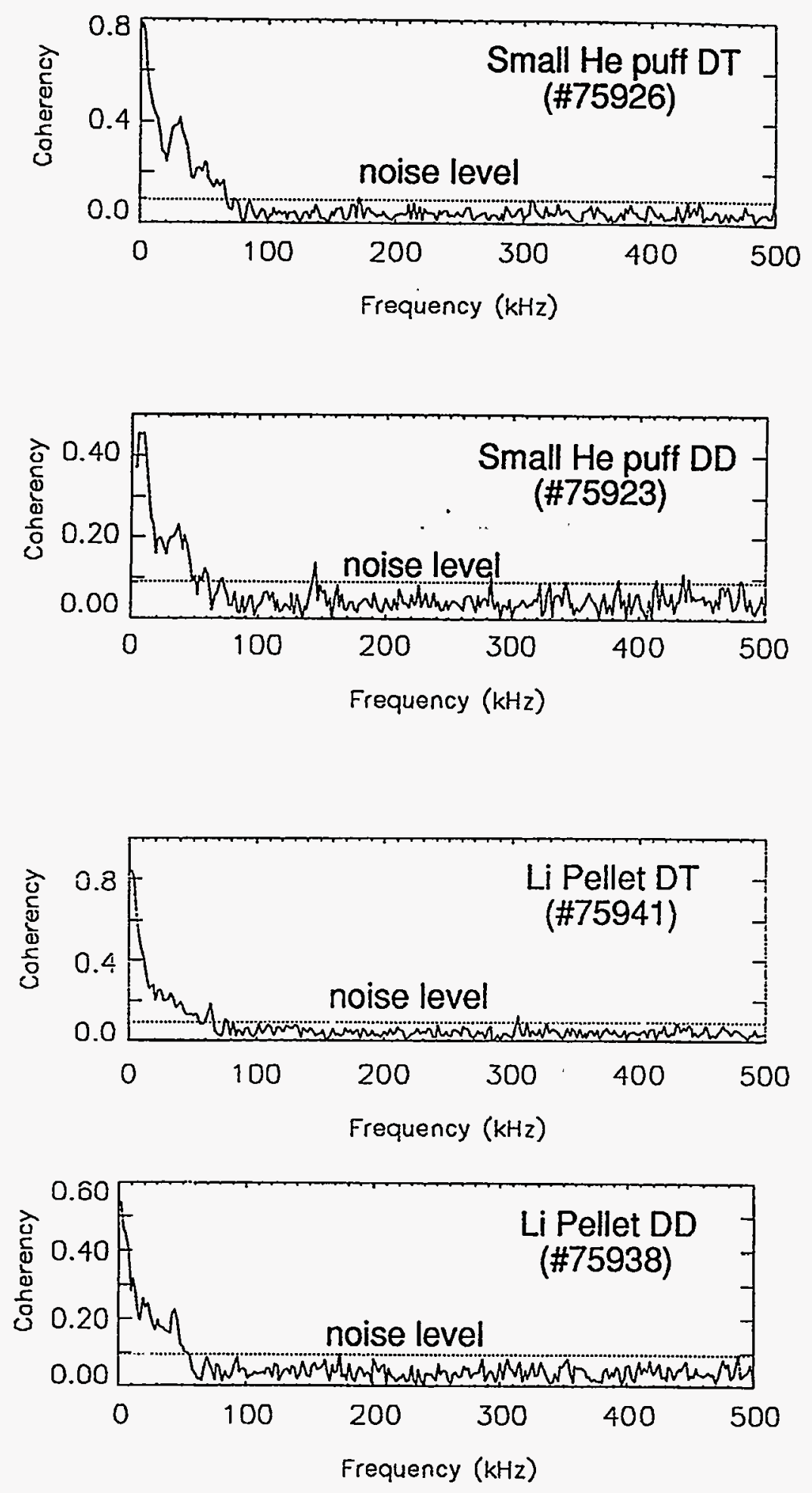

Fig. 12 

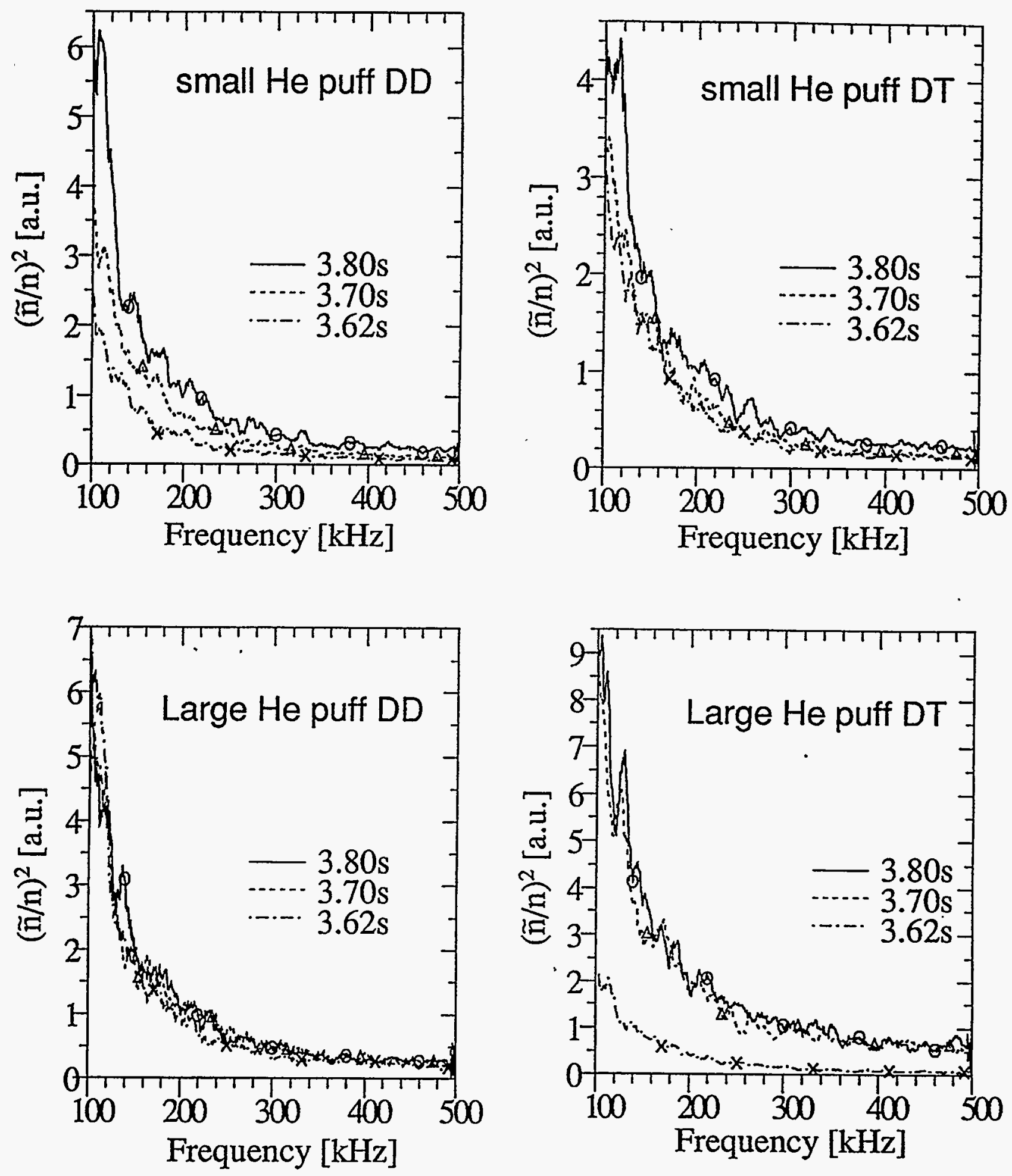

Fig. 13(a) 

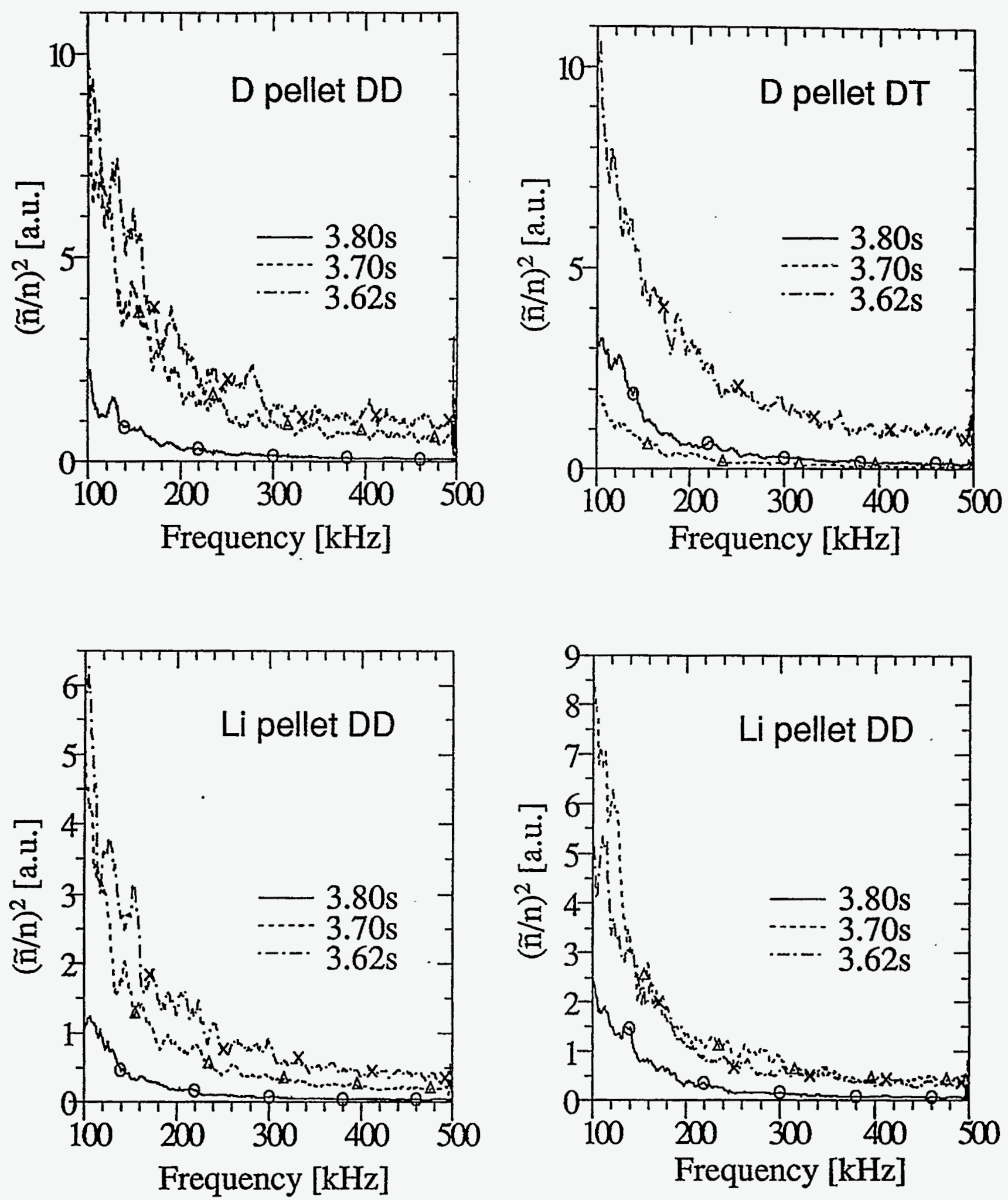

Fig. 13(b) 

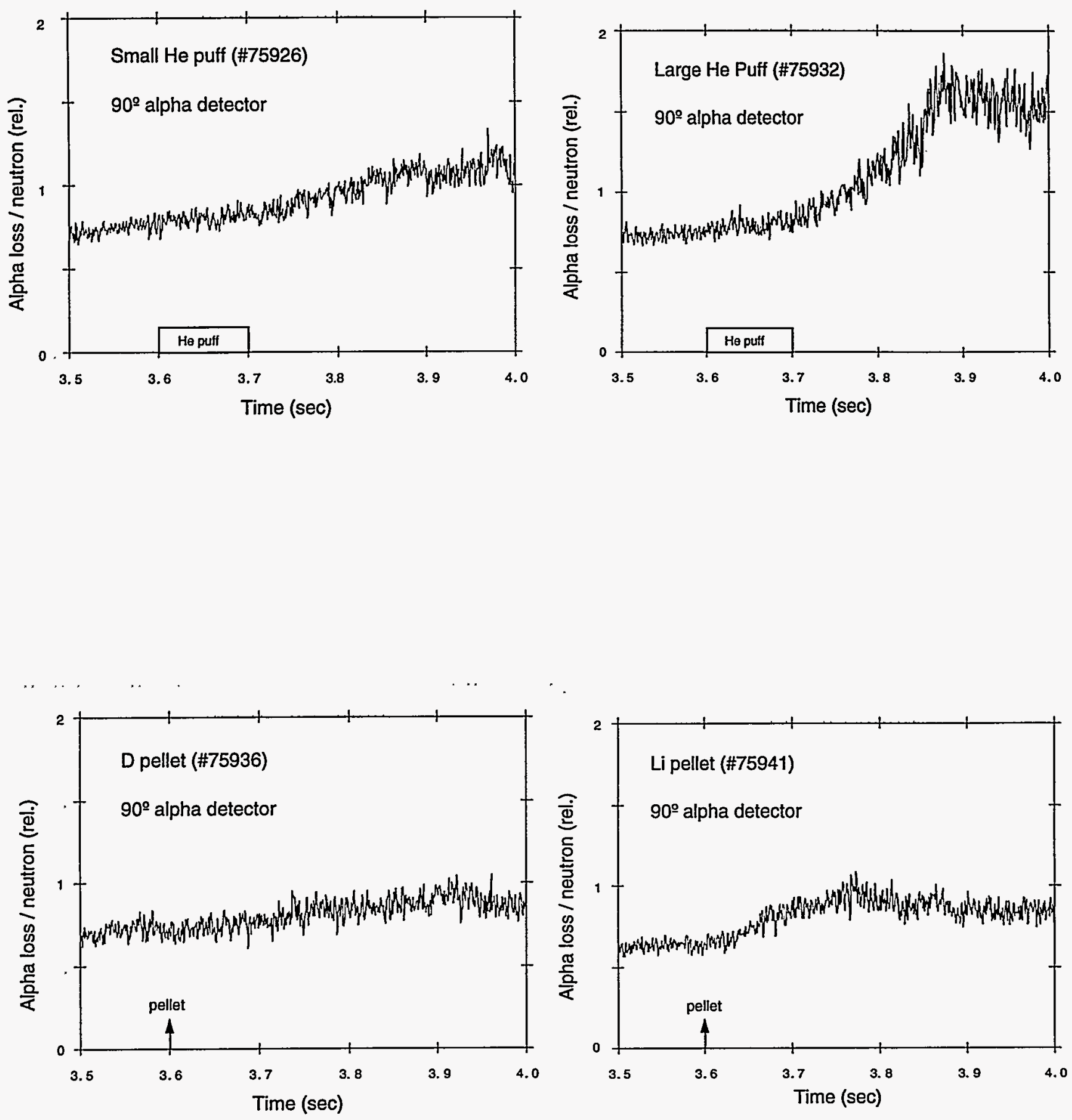

Fig. 14 


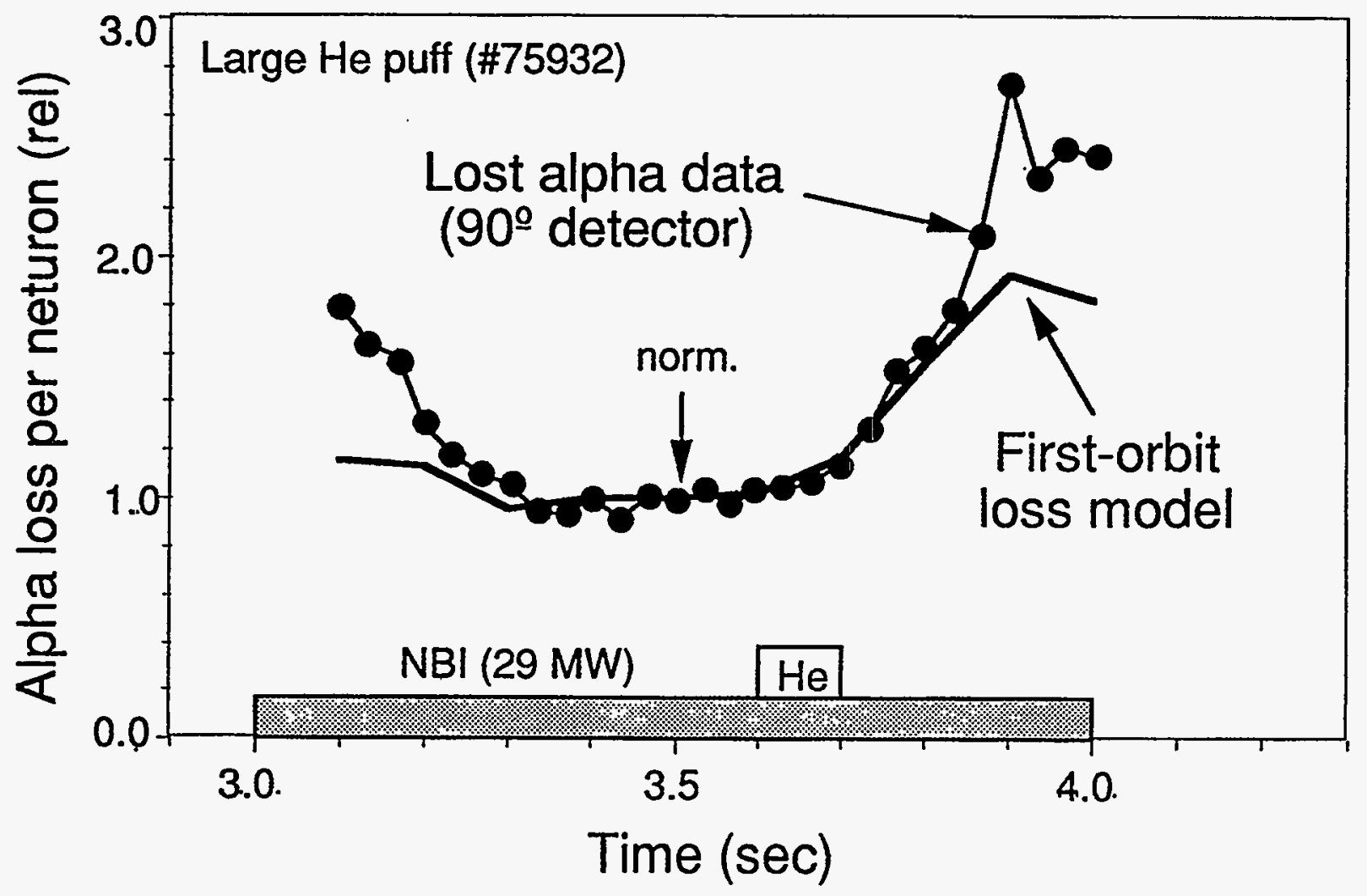

Fig. 15 

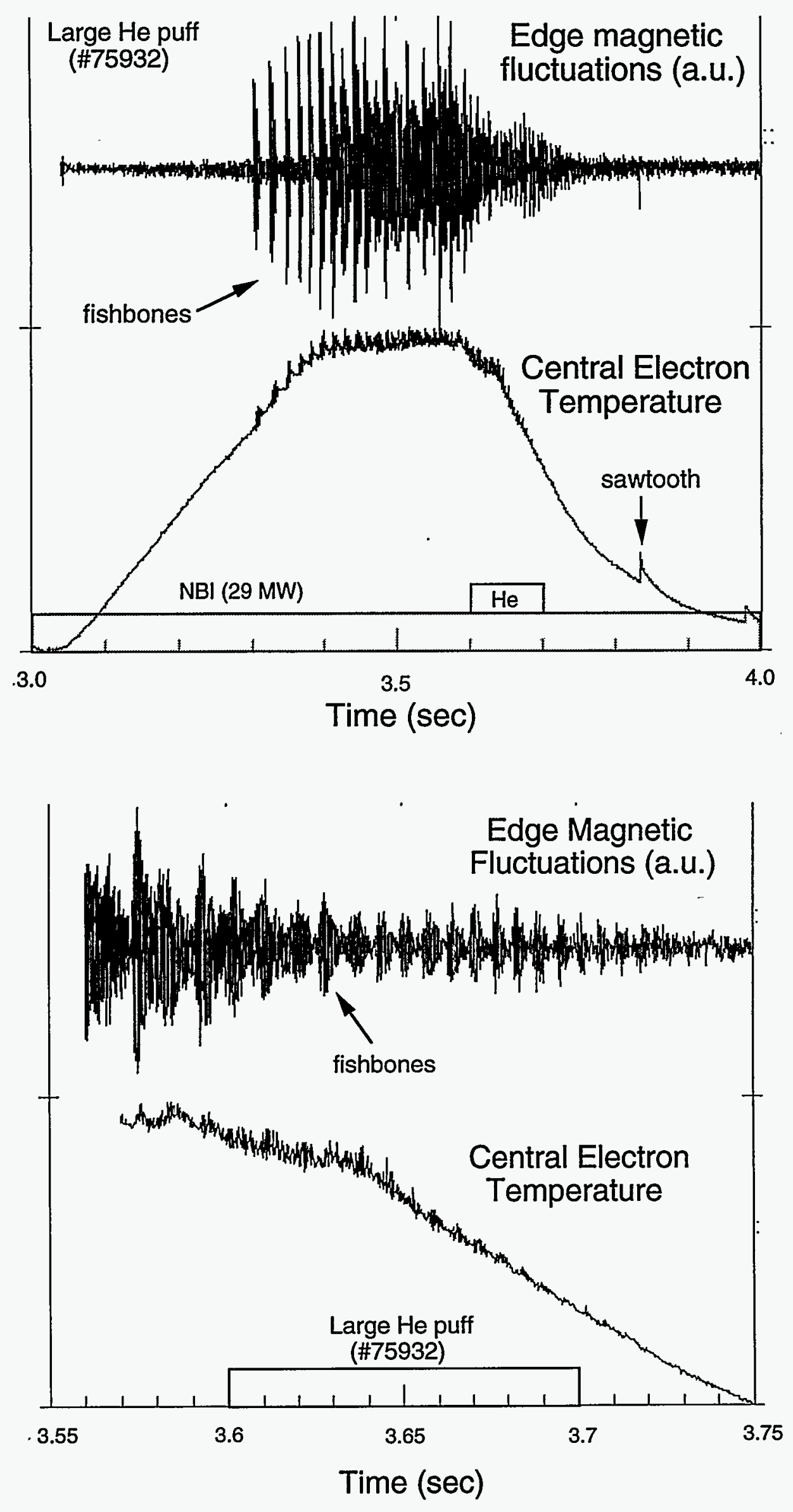

Fig. 16 

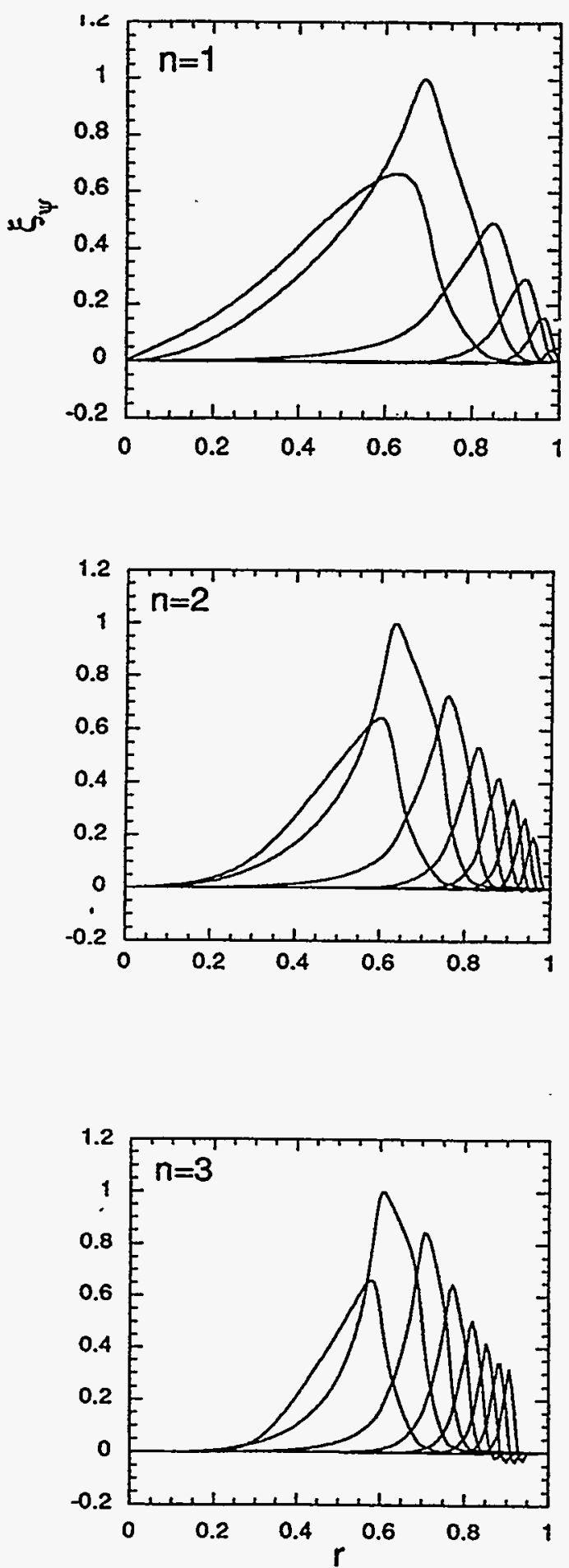

Fig. 17 

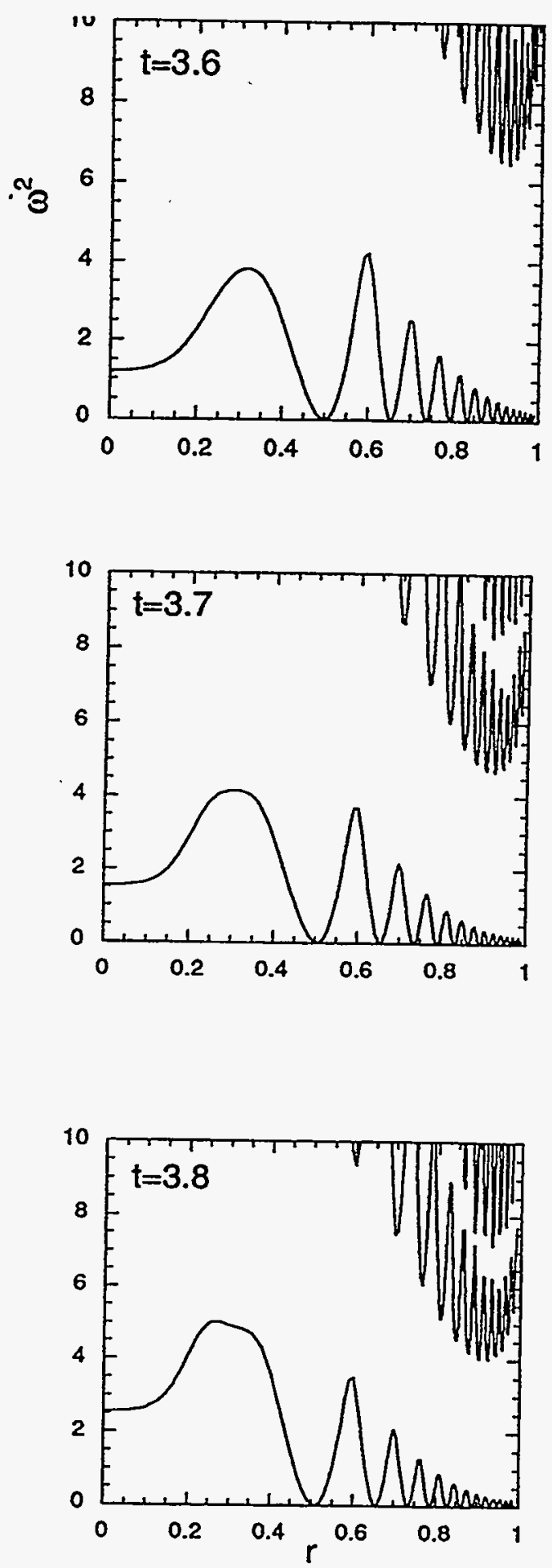

Fig. 18 


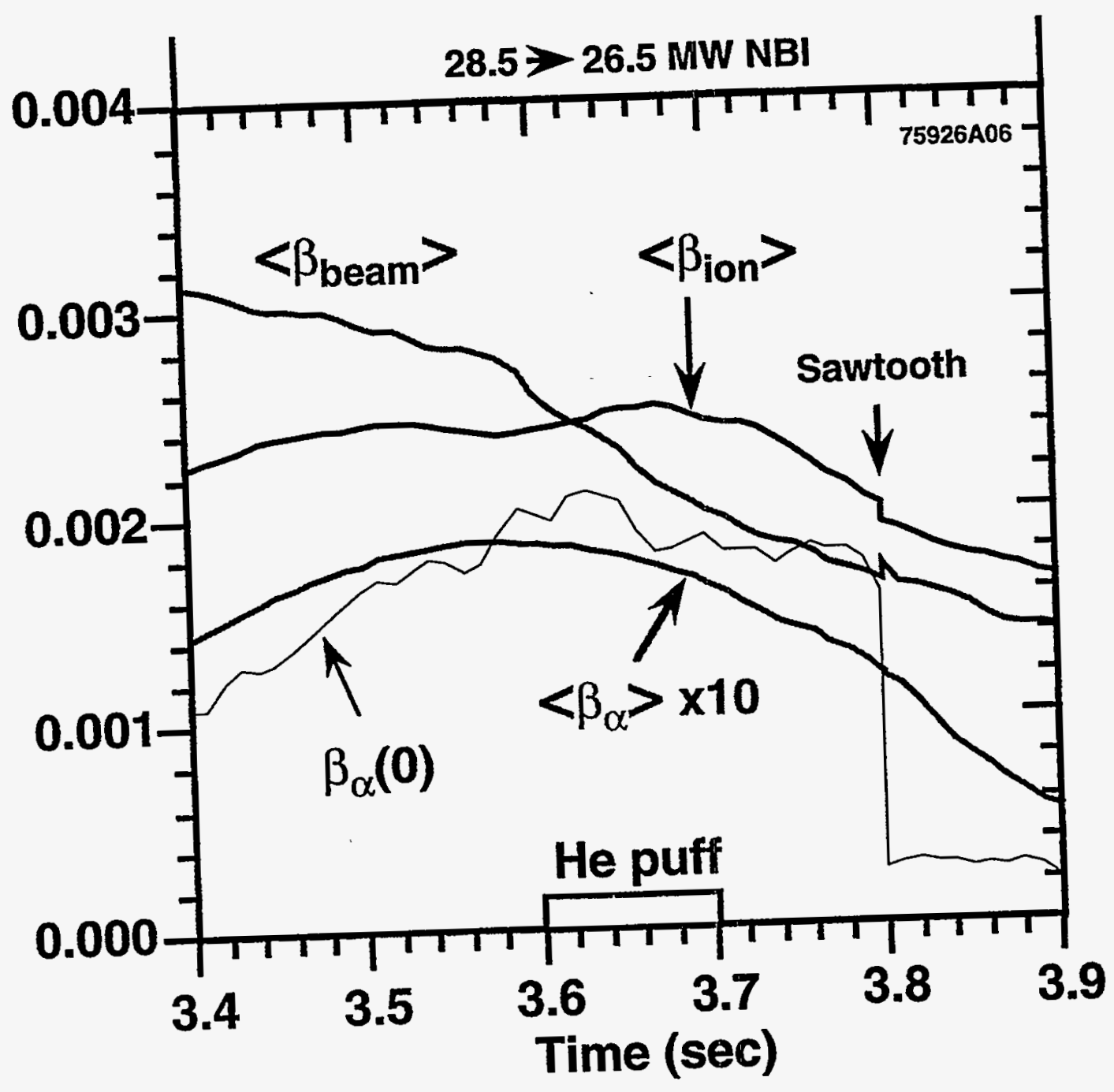

\section{DISCLAIMER}

This report was prepared as an account of work sponsored by an agency of the United States Government. Neither the United States Government nor any agency thereof, nor any of their employees, makes any warranty, express or implied, or assumes any legal liability or responsibility for the accuracy, completeness, or usefulness of any information, apparatus, product, or process disclosed, or represents that its use would not infringe privately owned rights. Reference herein to any specific commercial product, process, or service by trade name, trademark, manufacturer, or otherwise does not necessarily constitute or imply its endorsement, recommendation, or favoring by the United States Government or any agency thereof. The views and opinions of authors expressed herein do not necessarily state or reflect those of the United States Government or any agency thereof.

Fig. 19 

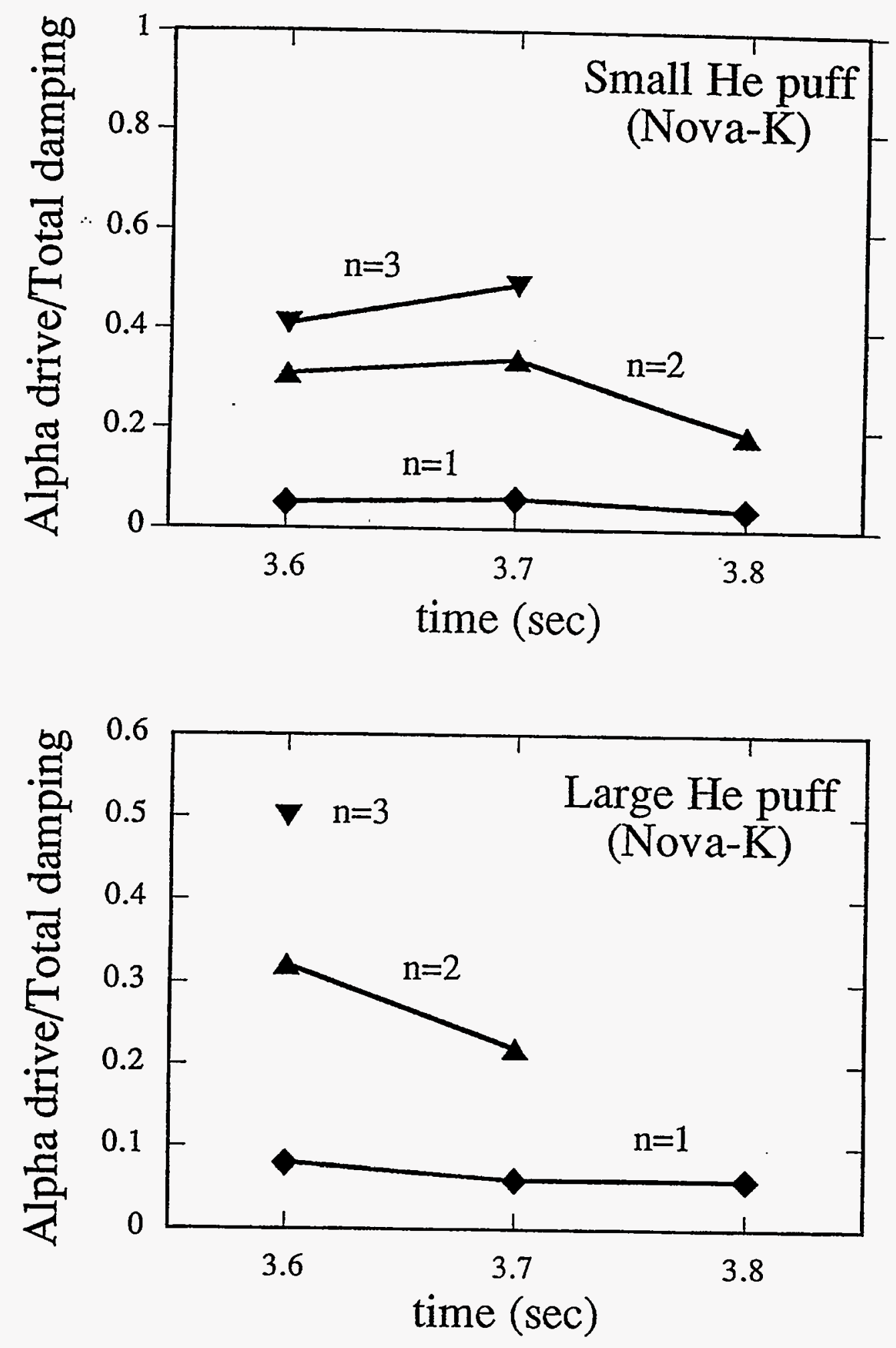

Fig. 20 

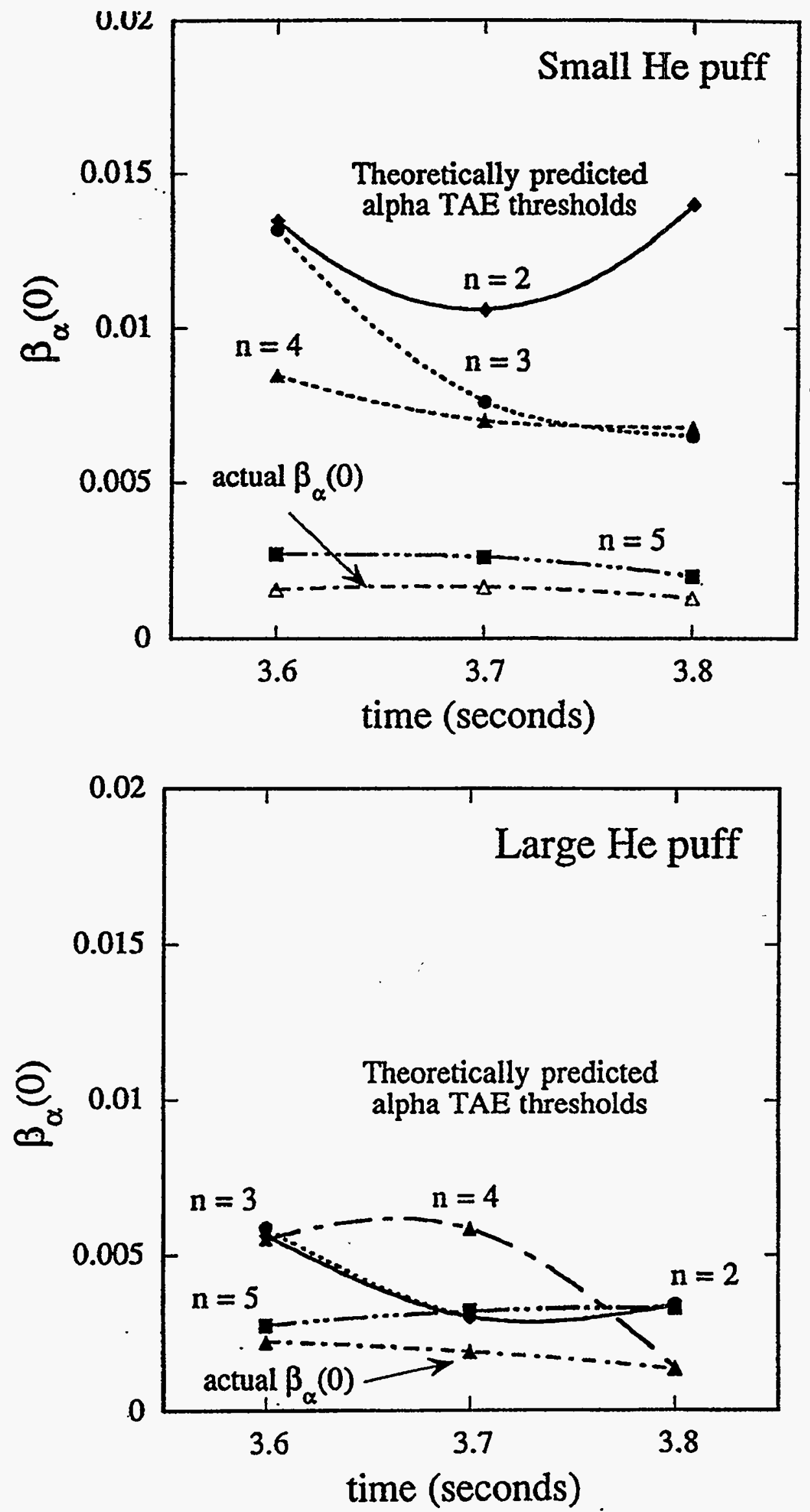

Fig. 21 